\title{
Strategic Voting over Strategic Proposals ${ }^{1}$
}

\author{
Philip Bond \\ Finance Department \\ The Wharton School \\ University of Pennsylvania \\ Philadelphia, PA 19104-6367 \\ pbond@wharton . upenn . edu
}

\author{
Hülya Eraslan \\ Finance Department \\ The Wharton School \\ University of Pennsylvania \\ Philadelphia, PA 19104-6367 \\ eraslan@wharton. upenn. edu
}

First draft: September 2004

This draft: January 2007

\footnotetext{
${ }^{1}$ We are grateful to Emeric Henry and Wolfgang Pesendorfer for incisive and very helpful comments on a prior draft. We thank John Duggan, Timothy Feddersen, Paolo Fulghieri, Andrew McLennan, Jean Tirole, Bilge Yllmaz, and audiences at the American Economic Association, the American Law and Economics Conference, the Federal Reserve Bank of Cleveland, Duke University, CEPR Gerzensee, the Laffont Memorial Conference, the Decentralization Conference in Paris, Northwestern University, the University of Oxford, the University of Pennsylvania, the Federal Reserve Bank of Philadelphia, the Texas Finance Festival, the Wallis Conference, and the World Congress of the Econometric Society for useful suggestions. Eraslan thanks the National Science Foundation and the Rodney White Center for financial support. Any remaining errors are our own.
} 


\begin{abstract}
Prior research on "strategic voting" has reached the conclusion that unanimity rule is uniquely bad: it results in destruction of information, and hence makes voters worse off. We show that this conclusion depends critically on the assumption that the issue being voted on is exogenous, i.e., independent of the voting rule used. We depart from the existing literature by endogenizing the proposal that is put to a vote, and establish that under many circumstances unanimity rule makes voters better off. Moreover, in some cases unanimity rule also makes the proposing individual better off, even when he has diametrically opposing preferences. In this case, unanimity is the Pareto dominant voting rule. Voters prefer unanimity rule because it induces the proposing individual to make a more attractive proposal. The proposing individual prefers unanimity rule because the acceptance probabilities for moderate proposals are higher.
\end{abstract}

JEL classification: C7; D7; D8.

Keywords: Strategic voting; agenda setting; multilateral bargaining. 


\section{Introduction}

Many collective decisions are made by holding a vote over an endogenously determined agenda. Examples include debt restructuring negotiations between a troubled company and its creditors; congressional votes over presidential appointments in the U.S. and elsewhere; shareholder votes on executive compensation; and collective bargaining between a firm and union members. The voting rules used for different decisions differ, and the choice of voting rule has two consequences. First, the voting rule affects whether a given proposal is adopted. Second, the voting rule affects the proposal that is being voted over.

A large and influential recent literature has analyzed voting when individuals have different information. ${ }^{1}$ This "strategic voting" literature has dealt exclusively with the first consequence of the voting rule - whether a given proposal is adopted — and reached the conclusion that unanimity rule is inferior to majority rule. ${ }^{2,3}$ Specifically, while majority rule aggregates information efficiently when the number of voters is large enough, unanimity rule always results in mistaken decisions. As such, when the issue being voted over is exogenous, unanimity rule is a suboptimal voting rule, and reduces the expected payoff of voting individuals.

Nevertheless, in practice unanimity rule is employed in many settings. For example, under the Trust Indenture Act of 1939 outstanding debt can be restructured only if all creditors agree. Likewise, promotion decisions in a number of professions require unanimous approval, as do the decisions of many international organizations. The

\footnotetext{
${ }^{1}$ See, for example, Austen-Smith and Banks (1996), Feddersen and Pesendorfer (1996, 1997, 1998), McLennan (1998), Duggan and Martinelli (2001), Doraszelski et al (2003), Persico (2004), Yariv (2004), Martinelli (2005), Meirowitz (2005), Gerardi and Yariv (forthcoming).

${ }^{2}$ By majority rule, we mean any threshold voting rule: that is, a proposal is accepted if the fraction voting to accept exceeds a pre-specified threshold.

${ }^{3}$ The main exception is Coughlan (2000), who shows that if pre-vote communication is possible and voter preferences are common knowledge and closely aligned, then both unanimity and majority rules may allow efficient aggregation of information. However, Austen-Smith and Feddersen (2006) show that if voter preferences are not common knowledge then unanimity is again the inferior voting rule from the perspective of information aggregation. Additionally, even Coughlan does not argue that unanimity rule is strictly superior to majority rule in the standard two-alternative voting game.
} 
results of the aforementioned voting literature suggest that a majority vote would be more efficient in such settings.

In this paper we show that the conclusion that majority rule is superior depends critically on the assumption that the proposal being voted over is exogenous. We do so by studying the second consequence of the voting rule mentioned above, namely that it affects the proposal being voted upon. We show that under many circumstances unanimity rule increases the expected utility of voting individuals, because it induces the proposing individual to make a more attractive offer. ${ }^{4}$ Further, in a subset of such circumstances unanimity rule is Pareto superior, because it also increases the proposing individual's expected utility — even though we model his interests as being diametrically opposed to those of the voting individuals.

Specifically, we consider the following setting. One individual — the proposer makes a take-it-or-leave-it offer to a large group. The group must collectively decide whether to accept or reject the offer, and we assume that it does so by holding a vote. For the remainder of the paper we refer to the group members as voters. The fraction of votes required to accept the proposer's offer is fixed prior to the offer (by, for example, law, contract, or the common consent of group members). As such, when the proposer makes his offer he takes the voting rule used by the group as given. The main assumption we make regarding preferences is that the set of feasible offers can be totally ordered, with voters preferring higher offers and the proposer preferring lower offers. That is, the proposer and voters have opposing interests, as is the case in many voting situations. We study the equilibrium payoffs when the number of voters is large.

As one would expect, and regardless of the voting rule, the acceptance probability is increasing in the attractiveness of the offer to voters. Consequently, the proposer faces a trade-off between a high offer that is accepted more often and a low offer that is accepted less often. Equilibrium offers are determined by this trade-off.

\footnotetext{
${ }^{4}$ For promotion decisions, the issue being voted upon is effectively the candidate's performance over the evaluation period, which is certainly endogenous.
} 
As in the prior literature, the group (asymptotically) makes the correct decision under majority rule but makes mistakes under unanimity rule. In particular, voters reject low offers more often than they should, and accept high offers more often than they should. Provided the proposer's payoff under disagreement is not too low, the mistakes that arise under unanimity rule benefit voters. In this case, when facing voters using majority rule, the proposer is not willing to make a high offer, but rather prefers a smaller offer accepted less often. Since voters make mistakes under unanimity rule by rejecting low offers more often than they should, the proposer needs to make an offer that is higher than he would make under majority rule.

Even though under unanimity rule voters receive a better offer from the proposer (provided the proposer's payoff under disagreement is not too low), it is still not obvious whether they prefer unanimity rule or majority rule. The reason is that the better offer is made as a direct consequence of voters' mistakes. However, we establish that a form of the envelope theorem holds in our voting environment. As such one can evaluate the effect of the higher offer simply by considering the direct effect, which is of course positive. It follows that voters' expected utility — as well as the equilibrium offer - is higher under unanimity rule.

Moreover, and surprisingly, when the proposer's payoff under disagreement is neither too low nor too high, the proposer also prefers unanimity rule, making it the Pareto dominant voting rule. The key to this result is that against a group using unanimity rule the proposer is able to get a moderate offer accepted with very high probability. In contrast, as described above the proposer's best offer against majority rule is a lower offer that is accepted with a lower probability. In this case voters prefer unanimity rule because they get a higher offer than they would under majority rule. The proposer prefers unanimity rule because he can secure acceptance more often than he could under majority rule at a cost he is willing to bear, due to the mistakes of the voters.

Overall, our results highlight the importance of the endogeneity of the proposal being 
voted over, i.e., the agenda, in the determination of optimal voting rules. While unanimity rule is inferior when the agenda is exogenous, it may Pareto dominate all other voting rules once the agenda is endogenous.

\section{RELATED Literature}

As discussed above, our paper develops the strategic voting literature by endogenizing the issue being voted over. This literature has studied how differentially informed individuals vote over an exogenously specified agenda by explicitly taking into account that a vote only matters if it is pivotal, and so each voter should condition on the information implied by being pivotal. In particular, it is not an equilibrium for each voter to vote sincerely, i.e., purely according to his own information (Austen-Smith and Banks 1996). When the number of voters is large, in equilibrium information is nonetheless aggregated efficiently under majority rule. In contrast, unanimity rule does not lead to efficient information aggregation, and therefore results in mistakes (see Feddersen and Pesendorfer 1997, 1998, and also Duggan and Martinelli 2001). Given these results, one might be tempted to conclude that unanimity rule is inefficient, and in particular, hurts voters. Our results show that neither is true when the agenda being voted on is endogenous.

Our paper is also related to the extensive recent literature on multilateral bargaining. Most of this literature has focused on proposals that can discriminate among individuals. ${ }^{5}$ However, in many negotiations a proposal must treat all members of some group equally, either for technological reasons (e.g., the building of a bridge), or for institutional/legal reasons (e.g., wage determination, debt restructuring).The literature analyzing this important class of bargaining problems is much smaller — see Banks and Duggan (2001), Cho and Duggan (2003), Cardona and Ponsati (2005), and Manzini and Mariotti (2005). These papers are deterministic complete information models, and as such, informational issues do not arise. Since agreement is always reached, there is no risk of breakdown of agreement from having a "tougher" bargaining stance. In contrast, the possibility

\footnotetext{
${ }^{5}$ The classic paper is Baron and Ferejohn (1989).
} 
of failing to agree to a Pareto improving proposal is central to our analysis and results. Chae and Moulin (2004) provide a family of solutions to group bargaining from an axiomatic viewpoint. Elbittar et al (2004) provide experimental evidence that the choice of voting rule used by a group in bargaining affects outcomes.

In our model, bargaining takes place under two-sided asymmetric information. The literature on bargaining under asymmetric information is extensive. ${ }^{6}$ We add to this literature by considering how the internal organization of one of the parties affects equilibrium outcomes.

Finally, in closely related independent work Henry (2006) instead takes the voting rule as fixed, and characterizes the proposer's best discriminatory offer. In other words, he studies the equilibrium proposals that emerge in the Baron-Ferejohn environment with asymmetric information.

\section{PAPER OUTLINE}

The paper proceeds as follows. In Section 2 we illustrate the main results and intuition through an example. We formalize and generalize this example in subsequent sections. Section 3 describes the model. Sections 4 and 5 establish equilibrium existence and characterize basic equilibrium properties. Section 6 bounds the equilibrium outcomes of the bargaining game when the group uses unanimity rule. Section 7 conducts the same exercise when the group adopts majority rule. Section 8 compares outcomes and payoffs from different voting rules. Section 9 concludes. All proofs are in the Appendix.

\section{An example}

To illustrate the main results of the paper, it is useful to consider the following example. A firm, which is initially wholly owned by a single individual (the debtor), seeks to restructure its outstanding debt by offering a group of creditors a share of its future cash

\footnotetext{
${ }^{6}$ See Kennan and Wilson (1993) for a review. Of most relevance for our paper are Samuelson (1984), Chatterjee and Samuelson (1987), Evans (1989), Vincent (1989), Schweizer (1989), and Deneckere and Liang (2006), all of which study common values environments.
} 
flow. If the creditors accept the offer, the debtor continues to run the firm - in which case he receives a utility equivalent to $\$ 120$, in addition to his share of firm cash flow. If instead creditors decline the offer, they liquidate the firm and obtain $\$ 100$, while the debtor receives nothing, but derives a utility of $\bar{V}<\$ 120$ from his outside option.

The future cash flow of the firm (if not liquidated) is uncertain: it is either $\$ 100$ or $\$ 200$, with ex ante equal probability. Each creditor possesses private information about the relative likelihood of the two valuations. To keep the example as transparent as possible, we assume the debtor has no private information about the future cash flow. We relax this assumption in our formal model below.

Assume that the number of creditors is large, and consider first the case in which the creditors use a majority rule. Since majority rule aggregates information efficiently ${ }^{7}$ the debtor's choice boils down to the following: either he can offer creditors $1 / 2$ of the future cash flow, so that they accept whenever the true cash flow of the firm is $\$ 200$; or he can offer creditors all the future cash flow, and gain acceptance all the time. Under the former alternative, the debtor's expected payoff is $\frac{1}{2}\left(\frac{1}{2} 200+120\right)+\frac{1}{2} \bar{V}$, while the latter alternative yields a payoff of 120. Consequently, if creditors use majority rule, the debtor offers all the firm's cash flow if his outside option is low enough - specifically, if $\bar{V}<20$ — and half of the cash flow otherwise. His expected utility is 120 in the former case, and $110+\frac{\bar{V}}{2}$ in the latter case, whereas creditors' expected payoff is $\frac{1}{2} 200+\frac{1}{2} 100=150$ in the former case, and $\frac{1}{2} \frac{1}{2} 200+\frac{1}{2} 100=100$ in the latter case.

Second, consider the case in which creditors instead use unanimity rule. Suppose also that the most negative signal, denoted $\underline{\sigma}$, received by an individual creditor is twice as likely to be received when the true cash flow is $\$ 100$ than when it is $\$ 200$, and so the probability of the $\$ 200$ cash flow conditional on signal $\underline{\sigma}$ is $1 / 3$. This implies that when the debtor offers $3 / 4$ of the cash flow to the creditors, there is an equilibrium in which all creditors vote to accept regardless of their signal. As such, under unanimity creditors mistakenly accept an offer of $3 / 4$ when the true cash flow is $\$ 100$. Formally, voting to

\footnotetext{
${ }^{7}$ See references above.
} 
accept the $3 / 4$ offer is a best response because when all other creditors vote to accept all the time, being pivotal does not convey any information. Given our assumption about the information content of the most negative signal $\underline{\sigma}$, conditional on all other creditors accepting, a creditor who observes $\underline{\sigma}$ is indifferent between accepting and rejecting the offer: if he accepts, the offer is accepted, and he expects to get

$$
\frac{1}{3} \times \frac{3}{4} \times 200+\frac{2}{3} \times \frac{3}{4} \times 100=100
$$

while if he rejects the offer is rejected and creditors get 100 in liquidation.

Given that creditors always accept an offer of $3 / 4$ of the cash flow, facing unanimity the debtor would not offer more than this amount. Moreover, given his outside option $\bar{V}<120$ he clearly prefers offering $3 / 4$ of the cash flow and gaining certain acceptance to offering nothing and ensuring certain rejection. Numerical simulations show that in this example the debtor's payoff is convex in his offer, ${ }^{8}$ and so the debtor's best offer under a unanimity voting rule is $3 / 4$. As a result, the debtor's expected payoff is $120+\frac{1}{2} 50+\frac{1}{2} 25=157.5$, whereas the creditors' unconditional expected payoff is $\frac{1}{2} 150+\frac{1}{2} 75=112.5$ for all $\bar{V}$.

Summarizing (see Figure 1), under majority rule, the debtor offers all the cash flow to the creditors if his utility from the outside option is low enough; otherwise, he offers half of the cash flow. When he offers all the cash flow, creditors accept with probability one, while when he offers half of the cash flow creditors accept only when the true cash flow is high. Under unanimity, regardless of his utility from the outside option, he offers a fraction $3 / 4$ of the cash flow to the creditors, and creditors mistakenly accept this offer all the time. It follows that, when the debtor's utility from his outside option is high enough $(\bar{V}>20)$, the creditors prefer unanimity, but when the debtor's utility from his outside option is low $(\bar{V}<20)$ the creditors prefer majority. In contrast, the debtor prefers unanimity when his utility from his outside option is low enough $(\bar{V}<95)$, and he prefers majority when his utility from his outside option is high $(\bar{V}>95)$.

\footnotetext{
${ }^{8}$ More generally, the proposing agent's payoff is convex in the offer whenever the signal quality of each voting agent is sufficiently small — see Lemma B-1 in Appendix B, available on the authors' webpages.
} 

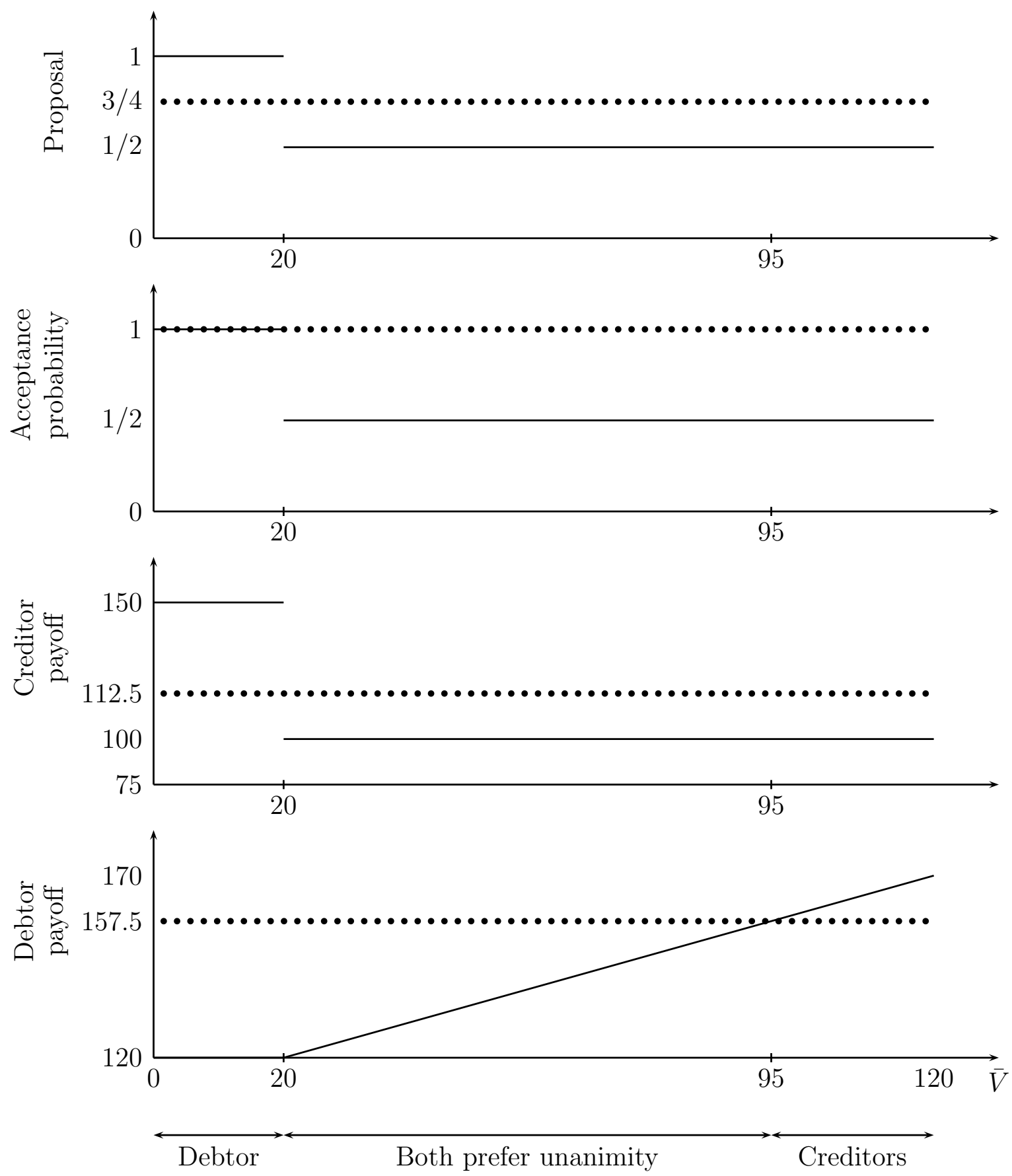

prefers unanimity

prefer unanimity

Figure 1: The graphs plot the equilibrium proposal, acceptance probability and expected payoffs. The solid lines correspond to majority rule while the dotted lines correspond to unanimity rule. 
Interestingly, it is possible that both the debtor and the creditors prefer unanimity rule to any majority rule. This happens when the debtor's outside option is intermediate $(20<\bar{V}<95)$. The creditors prefer unanimity rule because it delivers a higher offer relative to majority rule. The debtor prefers unanimity rule because he can get acceptance all the time at a smaller cost than he could under majority rule — the reason being that under unanimity rule, creditors mistakenly accept moderately high offers too often.

In the remainder of the paper we generalize this example. In particular, we:

- establish these results formally: A major (and unsatisfactory) shortcut in our presentation of the example is that we considered the offers made in response to the acceptance probabilities in an economy with infinitely many voters. Below, we instead first characterize the equilibrium set for a finite economy, and then take the limit. Doing so requires us to characterize the convergence properties of the acceptance probability function.

- establish these results for a fairly general specification of preferences: One property of preferences in the example is that the interests of creditors are completely aligned (pure common values). In our analysis we relax this assumption by working with preferences which allow for both private and common values. We establish all our results for preferences that are sufficiently close to pure common values. In doing so, we show that there is no discontinuity at the common values extreme.

- generalize the results to the case in which the proposing agent has some information: In the example, we assumed that the debtor has no information about the relative likelihood of the two states. In our analysis below we do not make this assumption. This introduces a signalling aspect to the game.

- establish that a generalized version of the envelope theorem holds in the voting game we analyze - even though the voting outcome reflects the decisions of many different voters. This step is necessary in order to show that voters prefer higher 
offers in spite of the changing incidence of mistakes.

\section{Model}

There is a single proposer (agent 0 ), and a group of $n \geq 2$ voters, labelled $i=1, \ldots, n$. The timing is as follows: (1) Each agent $i \in\{0,1, \ldots, n\}$ privately observes a random variable $\sigma_{i} \in[\underline{\sigma}, \bar{\sigma}]$. As we detail below, the realization of $\sigma_{i}$ affects agent $i$ 's preferences and/or information. (2) The proposer selects a proposal $x \in[0,1]$. (3) Voters simultaneously cast ballots to accept or reject the proposal. (4) If at least a fraction $\alpha$ of the voters accept ${ }^{9}$ the proposal is implemented, while otherwise the status quo prevails. Common examples include simple majority rule, $\alpha=1 / 2$; supermajority rule, e.g., $\alpha=2 / 3$; and unanimity rule, $\alpha=1$. We take the voting rule $\alpha$ to be exogenously given: ${ }^{10}$ in particular, it cannot be changed after the proposer makes his offer.

\section{PREFERENCES}

Agent $i$ 's preferences over the proposal $x$ and the status quo are determined by $\sigma_{i}$ and an unobserved state variable $\omega \in\{L, H\}$. The probability of state $\omega$ is $p^{\omega}$. We write voter $i$ 's utility associated with offer $x$ as $U^{\omega}\left(x, \sigma_{i}, \lambda\right)$, where $\lambda \in[0,1]$ is a parameter that describes the relative importance of $\omega$ and $\sigma_{i}$. We assume that $U^{\omega}\left(x, \sigma_{i}, \lambda\right)$ is independent of $\sigma_{i}$ at $\lambda=0$, and $U^{L}(\cdot, \cdot, \lambda) \equiv U^{H}(\cdot, \cdot, \lambda)$ at $\lambda=1$. Likewise, we write $\bar{U}^{\omega}\left(\sigma_{i}, \lambda\right)$ for voter $i$ 's utility under the status quo, and make parallel assumptions for $\lambda=0,1$. As such, our framework includes pure common values $(\lambda=0)$ and pure private values $(\lambda=1)$ as special cases. For the most part, we focus on preferences close to common values: many existing strategic voting papers deal exclusively with pure common values, ${ }^{11}$ and it is the natural benchmark in a variety of settings, e.g., debt

\footnotetext{
${ }^{9}$ Throughout, we ignore the issue of whether or not $n \alpha$ is an integer. This issue could easily be handled formally by replacing $n \alpha$ with $[n \alpha]$ everywhere, where $[n \alpha]$ denotes the smallest integer weakly greater than $n \alpha$. Since this formality has no impact on our results, we prefer to avoid the extra notation and instead proceed as if $n \alpha$ were an integer.

${ }^{10}$ Since our main results characterize the proposer's and voters preferences over different voting rules, it would be straightforward to endogenize the choice of voting rule by having either the voters or proposer select it at an ex ante stage before $\left\{\sigma_{i}\right\}$ are realized.

${ }^{11}$ See, for example, Feddersen and Pesendorfer (1998), and Duggan and Martinelli (2001).
} 
restructuring, where the securities received trade ex post.

A key object in our analysis is the utility of a voter from the proposal above and beyond the status quo. Accordingly, we define

$$
\Delta^{\omega}\left(x, \sigma_{i}, \lambda\right) \equiv U^{\omega}\left(x, \sigma_{i}, \lambda\right)-\bar{U}^{\omega}\left(\sigma_{i}, \lambda\right)
$$

Similarly, we write the proposer's utility from having his offer accepted as $V^{\omega}\left(x, \sigma_{0}\right)$, and his utility under the status quo as $\bar{V}^{\omega}\left(\sigma_{0}\right)$. Note that we do not require the relative weights of $\omega$ and $\sigma_{0}$ in determining the proposer's preferences to match the relative weights (given by $\lambda$ ) of $\omega$ and $\sigma_{i}$ in determining voter $i$ 's preferences.

For all preferences $\lambda<1$, the realization of $\sigma_{i}$ provides voter $i$ with useful (albeit noisy) information about the unobserved state variable $\omega$. We assume that the random variables $\left\{\sigma_{i}: i=0,1, \ldots, n\right\}$ are independent conditional on $\omega$, and that except for $\sigma_{0}$ (which is observed by the proposer) are identically distributed. Let $F(\cdot \mid \omega)$ and $F_{0}(\cdot \mid \omega)$ denote the distribution functions for the voters and proposer respectively. We assume that both distributions have associated continuous density functions, which we write $f(\cdot \mid \omega)$ and $f_{0}(\cdot \mid \omega)$. We let $\ell(\sigma)$ denote the likelihood ratio $\frac{f(\sigma \mid H)}{f(\sigma \mid L)}$, and $\ell_{0}(\sigma)$ denote the likelihood ratio $\frac{f_{0}(\sigma \mid H)}{f_{0}(\sigma \mid L)}$. The realization of $\sigma_{i}$ is informative about $\omega$, in the sense that the monotone likelihood ratio property (MLRP) holds strictly; ${ }^{12}$ but no realization is perfectly informative, i.e., $\ell(\underline{\sigma})>0$ and $\ell(\bar{\sigma})<\infty$, with similar inequalities for $\ell_{0}$. We denote the probability of state $\omega$ conditional on $\sigma_{i}$ by $p^{\omega}\left(\sigma_{i}\right)$.

\section{INTERPRETATIONS}

Possible interpretations of the model include the following:

(A) An indebted firm offers $n$ creditors an equity stake $x$ in exchange for the retirement of existing debt claims. If the creditors reject the offer the firm is liquidated. Let $\frac{1}{n} U^{\omega}\left(x, \sigma_{i}, \lambda\right)$ be the value of an $x / n$ share to creditor $i, \frac{1}{n} \bar{U}^{\omega}\left(\sigma_{i}, \lambda\right)$ be the value of receiving $1 / n$ of the liquidation value, ${ }^{13} V^{\omega}\left(x, \sigma_{0}\right)$ be the debtor's valuation of the

\footnotetext{
${ }^{12}$ That is, $\ell(\sigma)$ and $\ell_{0}(\sigma)$ are strictly increasing in $\sigma$.

${ }^{13}$ These preferences are isomorphic under any monotone transformation, and in particular, multiplication by $n$.
} 
remaining $1-x$ share if his offer is accepted, and $\bar{V}^{\omega}\left(\sigma_{0}\right)$ his payoff in liquidation.

(B) An employer is in wage negotiations with $n$ workers. He offers a wage $x$, which worker $i$ values at $U^{\omega}\left(x, \sigma_{i}, \lambda\right)$. If the offer is rejected, workers strike: $\bar{U}^{\omega}\left(\sigma_{i}, \lambda\right)$ is worker $i$ 's expected payoff from the strike. The firm's total profits if the offer is accepted are $n V^{\omega}\left(x, \sigma_{0}\right)$, and its expected total profits if a strike ensues are $n \bar{V}^{\omega}\left(\sigma_{0}\right)$.

(C) A president proposes a policy $x .^{14}$ The proposal is adopted only if passed by the legislature. This requires the support of a sufficient fraction of legislators from the opposing party to the president.

\section{EQUILIBRIUM}

We examine the sequential equilibria of the game just described. The proposer's strategy is a mapping from the set of possible signals, $[\underline{\sigma}, \bar{\sigma}]$, to probability distributions over the offer set $[0,1]$. Conditional on the proposer's offer, and as is standard in the strategic voting literature on which we build, we restrict attention to equilibria in which the ex ante identical voters behave symmetrically. ${ }^{15}$

Voters are potentially able to infer information about the proposer's observation of $\sigma_{0}$ from his offer, and thus information about the state variable $\omega$. Since only the latter affects voters' preferences, we focus directly on the beliefs about $\omega$ after observing an offer $x$. Let $\beta_{n}(x ; \lambda, \alpha)$ denote the voters' belief that $\omega=H$ after observing offer $x$ in the game with $n$ voters using voting rule $\alpha$, and preference parameter $\lambda$.

A sequential equilibrium thus consists of an offer strategy for the proposer, voter beliefs $\beta_{n}(\cdot ; \lambda, \alpha)$, and a voting strategy $[\underline{\sigma}, \bar{\sigma}] \rightarrow\{$ accept,reject $\}$ for each voter such that the proposer's strategy is a best response to voters' (identical) strategies; and each voter's strategy maximizes his expected payoff given beliefs $\beta_{n}(\cdot ; \lambda, \alpha)$ and all other voters use the same strategy; and the beliefs themselves are consistent. At a minimum, belief consistency requires that voters are never more (respectively, less) confident that the

\footnotetext{
${ }^{14} \mathrm{~A}$ judicial nominee, for example.

${ }^{15}$ Duggan and Martinelli (2001) give conditions under which the symmetric voting equilibrium is the unique equilibrium for unanimity rule.
} 
state is $H$ than the proposer himself is after he sees the most (respectively, least) pro- $H$ signal $\sigma_{0}=\bar{\sigma}$ (respectively, $\sigma_{0}=\underline{\sigma}$ ). That is, for all offers $x$,

$$
\frac{\beta_{n}(x ; \lambda, \alpha)}{1-\beta_{n}(x ; \lambda, \alpha)} \in\left[\ell_{0}(\underline{\sigma}) \frac{p^{H}}{p^{L}}, \ell_{0}(\bar{\sigma}) \frac{p^{H}}{p^{L}}\right] .
$$

Consequently consistency implies that $\beta_{n}(x ; \lambda, \alpha) \in[\underline{b}, \bar{b}]$, for some $0<\underline{b}<\bar{b}<1$.

Assumptions

Assumption $1 \Delta^{\omega}, V$ and $\bar{V}$ are twice continuously differentiable in their arguments.

Assumption $2 \Delta^{H} \geq \Delta^{L}$ and $\Delta^{\omega}$ is increasing in $\sigma_{i}$; both relations are strict for $x>0$.

Assumption 3 For all $\lambda, \Delta^{H}(0, \bar{\sigma}, \lambda)<0$ and $\Delta^{H}(1, \bar{\sigma}, \lambda)>0$.

Assumption 4 For all $x, V^{\omega}\left(x, \sigma_{0}\right)-\bar{V}^{\omega}\left(\sigma_{0}\right) \geq 0$ for $\omega=L, H$ and all $\sigma_{0}$.

Assumption $5 \Delta^{\omega}$ is strictly increasing and $V$ is strictly decreasing in $x$.

Assumption 1 is standard. For future reference, observe that $\left|\Delta^{\omega}\right|$ is bounded above since $\Delta^{\omega}$ is continuous in its arguments and has compact domain.

Assumption 2 says voter $i$ is more pro-acceptance when $\omega=H$ than $\omega=L$, and when the realization of $\sigma_{i}$ is higher. Since higher values of $\sigma_{i}$ are more likely when $\omega=$ $H$ (by MLRP), the content of Assumption 2 (beyond being a normalization) is that the "private" and "common" components of voter utility act in the same direction.

Assumption 3 says that the voters regard the worst offer $(x=0)$ as worthless, i.e., they prefer the status quo. On the other hand, there are some offers which the voters view as worthwhile under some conditions - in particular, voter $i$ prefers the best offer $(x=1)$ to the status quo when $\omega=H$ and $\sigma_{i}=\bar{\sigma}$.

Assumption 4 says that the proposer strongly dislikes the status quo relative to the range of possible alternatives: regardless of the state, he would prefer to have any proposal $x \in[0,1]$ implemented. ${ }^{16}$

\footnotetext{
${ }^{16}$ In general, one can clearly think of a broader range of proposals $[0, \infty)$, but with the proposer preferring the status quo to offers $x \in(1, \infty)$. The content of Assumption 4 is that $x=1$ is the highest offer the proposer is prepared to make for any pair $\left(\omega, \sigma_{0}\right)$. For instance, in our debt renegotiation example, the debtor (the proposer) prefers being left with any fraction $1-x$ of the firm to liquidation.
} 
Finally, Assumption 5 says that the proposer and voters have diametrically opposing preferences: higher $x$ makes the voters more pro-agreement, but reduces the proposer's payoff if his proposal is accepted.

Before turning to the analysis of our model, we note that in our framework voting is the only means by which voters can share their information. When voters are numerous and dispersed, as is often the case, this is a reasonably realistic assumption. We return to this issue in more detail in the conclusion. Somewhat related, we also take as given the information possessed by voters. Other authors have modelled strategic voting games with costly information acquisition, ${ }^{17}$ but have done so under the assumption that the proposal being voted over is independent of the voting rule, i.e., is exogenous. We leave the simultaneous integration of costly information acquisition and endogenous proposals into strategic voting for future work.

\section{Equilibrium characterization and existence}

In this section we establish equilibrium existence, along with a number of characterization results. We first look at voting stage of the game.

\section{The Voting STAGE}

Fix a preference parameter $\lambda$ and a number of voters $n$. Having observed the proposer's offer $x$, each voter attaches a subjective probability $b=\beta_{n}(x ; \lambda, \alpha)$ to the state variable $\omega$ being $H$. A central insight of the existing strategic voting literature is that voter $i$ 's voting decision depends on the comparison of his expected utilities from accepting and rejecting, conditional on the event of being pivotal. Taking the strategies of other voters as given, let $P I V$ denote the event that his vote is pivotal. Thus voter $i$ votes to accept offer $x$ after observing $\sigma_{i}$ if and only if

$$
\operatorname{Pr}_{b}\left(H \mid P I V, \sigma_{i}\right) \Delta^{H}\left(x, \sigma_{i}, \lambda\right)+\operatorname{Pr}_{b}\left(L \mid P I V, \sigma_{i}\right) \Delta^{L}\left(x, \sigma_{i}, \lambda\right) \geq 0
$$

\footnotetext{
${ }^{17}$ See Persico (2004), Yariv (2004), and Martinelli (2005).
} 
where $\operatorname{Pr}_{b}$ denotes the subjective probability given $b$. Observe that even though voter $i$ does not observe $\sigma_{j}(j \neq i)$, and does not know whether or not he is actually pivotal, in casting his vote he considers only the payoffs in events in which he is pivotal, and takes into account any information he can thus infer.

Since the random variables $\sigma_{i}$ are independent conditional on $\omega$,

$$
\operatorname{Pr}_{b}\left(\omega \mid P I V, \sigma_{i}\right)=\frac{\operatorname{Pr}_{b}\left(\omega, P I V, \sigma_{i}\right)}{\operatorname{Pr}_{b}\left(P I V, \sigma_{i}\right)}=\frac{\operatorname{Pr}(P I V \mid \omega) \operatorname{Pr}\left(\sigma_{i} \mid \omega\right) \operatorname{Pr}_{b}(\omega)}{\operatorname{Pr}_{b}\left(P I V, \sigma_{i}\right)}
$$

Substituting (3) into inequality (2), and noting that $\operatorname{Pr}_{b}(H)=b=1-\operatorname{Pr}_{b}(L)$, voter $i$ votes to accept proposal $x$ after observing $\sigma_{i}$ if and only if

$$
\Delta^{H}\left(x, \sigma_{i}, \lambda\right) \operatorname{Pr}(P I V \mid H) f\left(\sigma_{i} \mid H\right) b+\Delta^{L}\left(x, \sigma_{i}, \lambda\right) \operatorname{Pr}(P I V \mid L) f\left(\sigma_{i} \mid L\right)(1-b) \geq 0
$$

By MLRP, it is immediate from (4) that in any equilibrium each voter $i$ follows a cutoff strategy, in the sense of voting to accept if and only if $\sigma_{i}$ exceeds some critical level. As noted, throughout we focus on symmetric equilibria in which the ex ante identical voters follow the same voting strategy. Let $\sigma_{n}^{*}(x, b, \lambda, \alpha) \in[\underline{\sigma}, \bar{\sigma}]$ denote the common cutoff ${ }^{18}$ when there are $n$ voters, the offer is $x$, voters attach a probability $b$ to $\omega=H$, and the preference parameter and voting rule are $\lambda$ and $\alpha$ respectively. For clarity of exposition, we will suppress the arguments $n, x, b, \lambda$ and $\alpha$ unless needed, both for $\sigma^{*}$ and all other variables introduced below.

Evaluating explicitly, the probability that a voter is pivotal is given by

$$
\operatorname{Pr}(P I V \mid \omega)=\left(\begin{array}{c}
n-1 \\
n \alpha-1
\end{array}\right)\left(1-F\left(\sigma^{*}(x) \mid \omega\right)\right)^{n \alpha-1} F\left(\sigma^{*}(x) \mid \omega\right)^{n-n \alpha} .
$$

The acceptance condition (4) then rewrites to:

$$
\begin{aligned}
& \Delta^{H}\left(x, \sigma_{i}, \lambda\right)\left(1-F\left(\sigma^{*}(x) \mid H\right)\right)^{n \alpha-1} F\left(\sigma^{*}(x) \mid H\right)^{n-n \alpha} f\left(\sigma_{i} \mid H\right) b \\
& +\Delta^{L}\left(x, \sigma_{i}, \lambda\right)\left(1-F\left(\sigma^{*}(x) \mid L\right)\right)^{n \alpha-1} F\left(\sigma^{*}(x) \mid L\right)^{n-n \alpha} f\left(\sigma_{i} \mid L\right)(1-b) \geq 0
\end{aligned}
$$

If there exists a $\sigma^{*} \in[\underline{\sigma}, \bar{\sigma}]$ such that voter $i$ is indifferent between accepting and rejecting the offer $x$ exactly when he observes the signal $\sigma_{i}=\sigma^{*}$, then the equilibrium can be said

\footnotetext{
${ }^{18}$ As we show below, there exists a unique cutoff signal.
} 
to be a responsive equilibrium. Notationally, we represent a responsive equilibrium by its corresponding cutoff value $\sigma^{*} \in[\underline{\sigma}, \bar{\sigma}]$.

For use below, define the function

$Z(x, \sigma, b, \lambda, \alpha, n) \equiv b \Delta^{H}(x, \sigma) \ell(\sigma)\left(\frac{F(\sigma \mid H)}{F(\sigma \mid L)}\right)^{n-n \alpha}\left(\frac{1-F(\sigma \mid H)}{1-F(\sigma \mid L)}\right)^{n \alpha-1}+(1-b) \Delta^{L}(x, \sigma)$.

If $Z(x, \sigma)$ is positive (negative), and all but one of the voters use a cutoff strategy $\sigma$, then the remaining voter $i$ is better off voting to accept (reject) the proposal $x$ if he observes $\sigma_{i}=\sigma$. Similarly, if $Z(x, \sigma)=0$ then there is a responsive equilibrium in which all voters use the cutoff strategy $\sigma$.

By the Theorem of the Maximum, $\max _{\sigma \in[\underline{\sigma}, \bar{\sigma}]} Z(x, \sigma)$ and $\min _{\sigma \in[\underline{\sigma}, \bar{\sigma}]} Z(x, \sigma)$ are both continuous in $x$. So we can define $\underline{x}_{n}(b, \lambda, \alpha)$ and $\bar{x}_{n}(b, \lambda, \alpha)$ that describe the range of offers for which a responsive equilibrium exists: ${ }^{19}$

$$
\begin{aligned}
& \underline{x}_{n}(b, \lambda, \alpha)= \begin{cases}\min \left\{x \mid \max _{\sigma} Z(x, \sigma) \geq 0\right\} & \text { if }\left\{x \mid \max _{\sigma} Z(x, \sigma) \geq 0\right\} \neq \varnothing \\
1 & \text { otherwise }\end{cases} \\
& \bar{x}_{n}(b, \lambda, \alpha)= \begin{cases}\max \left\{x \mid \min _{\sigma} Z(x, \sigma) \leq 0\right\} & \text { if }\left\{x \mid \min _{\sigma} Z(x, \sigma) \leq 0\right\} \neq \varnothing \\
0 & \text { otherwise }\end{cases}
\end{aligned}
$$

That is, $\underline{x}_{n}(b, \lambda, \alpha)$ is the lowest offer that is ever accepted in a responsive equilibrium: if $x<\underline{x}_{n}(b, \lambda, \alpha)$, then $Z(x, \sigma)<0$ for all $\sigma$. Similarly, $\bar{x}_{n}(b, \lambda, \alpha)$ is the highest offer that is ever rejected in a responsive equilibrium.

The following lemma establishes existence and uniqueness of cutoff strategies in the voting stage of the game. Part (1) extends Theorem 1 of Duggan and Martinelli (2001) to our more general preference framework. Parts (2) and (3) establish elementary properties of how the responsive equilibrium is related to the proposer's offer $x$.

Lemma 1 (Existence and uniqueness in the voting stage) Fix beliefs b, a voting rule $\alpha$ and preferences $\lambda$. Then:

\footnotetext{
${ }^{19}$ Observe that $\underline{x}_{n}(b, \lambda, \alpha)>0$ since, by Assumptions 2 and $3, Z(0, \sigma)<0$ for all $\sigma$.
} 
(1) For any $n$, a responsive equilibrium $\sigma^{*}(x) \in[\underline{\sigma}, \bar{\sigma}]$ exists if and only if $x \in\left[\underline{x}_{n}, \bar{x}_{n}\right]$. When a responsive equilibrium exists it is the unique symmetric responsive equilibrium. (2) The equilibrium cutoff $\sigma^{*}(x)$ is decreasing and continuously differentiable over $\left(\underline{x}_{n}, \bar{x}_{n}\right)$, with $\sigma^{*}\left(\underline{x}_{n}\right)=\bar{\sigma}$ and $\sigma^{*}\left(\bar{x}_{n}\right)=\underline{\sigma}$.

(3) If $\alpha<1$ and $x$ is such that $\Delta^{H}(x, \bar{\sigma})>0>\Delta^{L}(x, \underline{\sigma})$, there exists $N$ such that $x \in\left(\underline{x}_{n}, \bar{x}_{n}\right)$ for $n \geq N$.

In addition to responsive equilibria, non-responsive equilibria exist. Specifically, for any $\alpha>\frac{1}{n}$ there is an equilibrium in which each voter rejects regardless of his signal, i.e., $\sigma^{*}=\bar{\sigma}$. Likewise, for any $\alpha<1-\frac{1}{n}$ there is an equilibrium in which each voter accepts regardless of his signal, i.e., $\sigma^{*}=\underline{\sigma}$. We follow the literature and assume that if a responsive equilibrium exists, then it is played. From Lemma 1, as $x$ increases over the interval $\left(\underline{x}_{n}, \bar{x}_{n}\right)$ the acceptance probability increases continuously from 0 to 1 . We thus assume that when $x \leq \underline{x}_{n}$ the rejection equilibrium is played, while for $x \geq \bar{x}_{n}$ the acceptance equilibrium is played. In addition to being intuitive and ensuring continuity, this rule selects the unique trembling-hand perfect equilibrium when $x \leq \underline{x}_{n}{ }^{20}$

\section{EQUILIBRIUM EXISTENCE}

In our environment, the proposer chooses an offer $x$ from an infinite choice set $[0,1]$. Additionally, the proposer "type" $\sigma_{0}$ is itself drawn from an infinite set $[\underline{\sigma}, \bar{\sigma}]$. It is well-known that sequential equilibria may fail to exist in infinite games, even when (as is the case here) payoff functions are continuous.

To establish equilibrium existence, we exploit Manelli's (1996) sufficient conditions for equilibrium existence in a canonical signaling game, in which a single "sender" of

\footnotetext{
${ }^{20}$ Formally, for any beliefs $b$, preference parameter $\lambda$ and voting rule $\alpha>\frac{1}{2}+\frac{1}{2 n}$, if $x \leq \underline{x}_{n}$ then the only trembling-hand perfect equilibrium is the non-responsive equilibrium in which each voter always rejects. A proof is available upon request.

Moreover, although when $x \geq \bar{x}_{n}$ both the acceptance and rejection equilibria are trembling-hand perfect, the trembles required to support the rejection equilibrium do not satisfy the cutoff rule property we discussed earlier. Indeed, if tremble strategies were required to satisfy the mild monotonicity restriction that voting to accept is weakly more likely after a higher signal, then the acceptance equilibrium would be the only trembling-hand perfect equilibrium when $x \geq \bar{x}_{n}$.
} 
unknown type chooses an action, and a single uninformed "receiver" selects a response. To apply his results, we must first show that the aggregate behavior of the $n$ partially informed voters in our model matches that of a single uninformed receiver endowed with suitable preferences. The following result does just this:

Lemma 2 (Equivalent sender-receiver game) Fix $n, \lambda$, $\alpha$. Suppose that the proposer makes an offer $x$ and the voters' beliefs about the proposer's observation $\sigma_{0}$ are given by the probability distribution $\varphi$ on $[\underline{\sigma}, \bar{\sigma}]$. Then the equilibrium $\sigma_{n}^{*}$ of the voting stage of the game coincides with the best-response correspondence of a single fictitious player holding the same beliefs and whose payoff depends on the offer $x$, proposer signal $\sigma_{0}$, and his own action $\sigma^{\prime}$ according to

$$
U_{n}\left(x, \sigma^{\prime}, \sigma_{0} ; \lambda, \alpha\right) \equiv \int_{\underline{\sigma}}^{\sigma^{\prime}}-Z\left(x, s, b=p^{H}\left(\sigma_{0}\right), \lambda, \alpha, n\right) d s .
$$

Our model is a stylized bargaining model in which an opposing party makes take-itor-leave-it offers to a group. In practice, there are many instances in which a group of individuals is engaged in collective bargaining. In such instances, it is often tempting to model the group as a single individual. Lemma 2 suggests that to some extent this approach is viable, but that the relation between the true preferences of individuals and those of the "representative" agent may be quite complicated. In particular, the utility function defined by (9) does not equal the average expected utility of a voter in the model

From Lemma 2, Manelli's results immediately imply:

Proposition 1 (Equilibrium existence) An equilibrium exists.

\section{$5 \quad$ Equilibrium properties}

We next establish general properties of equilibrium strategies and payoffs that we will use in our comparison of voting rules later in the paper. In particular, we show that a higher offer increases the expected utility of voters, even though a higher offer also changes the incidence of voting mistakes. 
We start with the following straightforward corollary to Lemma 1:

Corollary 1 (Change in offer and voter beliefs) The acceptance probability is continuous and monotonically increasing in the offer $x$ and voter beliefs $b$.

The heart of our analysis concerns the effect of the voting rule on the proposer's offer $x$, and in turn the effect on voter and proposer payoffs. Notationally, we write $\Pi_{n}^{V}(x, b, \lambda, \alpha)$ for a voter's expected payoff from offer $x$ under voting rule $\alpha$, voter preferences $\lambda$, and voter beliefs $b$; and $\Pi_{n}^{P}\left(x, \sigma_{0}, b, \lambda, \alpha\right)$ for the proposer's expected payoff after observing $\sigma_{0}$. Before proceeding, we note a second straightforward corollary of Lemma 1 :

Corollary 2 (Continuity and differentiability of payoffs) $\Pi_{n}^{V}(x, b, \lambda, \alpha)$ and $\Pi_{n}^{P}\left(x, \sigma_{0}, b, \lambda, \alpha\right)$ are continuous functions of the offer $x$, and are differentiable except at the boundaries of the responsive equilibrium range, $\underline{x}_{n}(b, \lambda, \alpha)$ and $\bar{x}_{n}(b, \lambda, \alpha)$.

One way that voting rules affect payoffs is through their impact on the equilibrium offer. As such, it is important to characterize how the voter payoff $\Pi_{n}^{V}$ depends on $x$. The main complication in doing so is that as $x$ changes the equilibrium of the voting stage changes, and so the standard envelope theorem does not apply. However, the envelope theorem can be adapted as follows.

Notationally, for an arbitrary profile of voter cutoff voting strategies $\hat{\sigma}_{1}, \ldots, \hat{\sigma}_{n}$, define $u_{i}\left(x, \hat{\sigma}_{1}, \ldots, \hat{\sigma}_{n}, b, \lambda, \alpha\right)$ as the expected payoff of voter $i$ given offer $x$. Write $u_{i}(x, \hat{\sigma}, b, \lambda, \alpha)$ for the special case in which all voters use the same strategy. Suppose for now that the voting equilibrium is responsive. Evaluating the effect of the offer $x$ on voter payoffs $\Pi_{n}^{V}$ explicitly,

$$
\frac{\partial}{\partial x} \Pi_{n}^{V}(x, b, \lambda, \alpha)=\frac{\partial}{\partial x} u_{i}\left(x, \sigma_{n}^{*}(x), b, \lambda, \alpha\right)+\sum_{j=1}^{n} \frac{\partial \sigma_{n}^{*}(x)}{\partial x} \frac{\partial}{\partial \hat{\sigma}_{j}} u_{i}\left(x, \sigma_{n}^{*}(x), b, \lambda, \alpha\right) .
$$

As in the standard envelope theorem, the fact that $\sigma_{n}^{*}(x)$ is an equilibrium strategy implies that

$$
\frac{\partial}{\partial \hat{\sigma}_{j}} u_{j}\left(x, \sigma_{n}^{*}(x), b, \lambda, \alpha\right)=0
$$


for all $j, x, b, \lambda$, and $\alpha$. In the pure common values setting $(\lambda=0) u_{j} \equiv u_{i}$, and so

$$
\frac{\partial}{\partial \hat{\sigma}_{j}} u_{i}\left(x, \sigma_{n}^{*}(x), b, \lambda, \alpha\right)=0
$$

for all $i, j, x, b, \lambda, \alpha$. So in the pure common values case, one can evaluate the effect of a change in the offer $x$ on voter payoffs simply by looking at the direct effect on utility (evaluated at the best response to the offer) - exactly as in the envelope theorem. Moreover, when voters use unanimity rule this argument extends to arbitrary preferences:

Proposition 2 (Effect of higher offers on voter payoffs) If $\alpha=1$ or $\lambda=0$,

$$
\frac{\partial}{\partial x} \Pi_{n}^{V}(x, b, \lambda, \alpha) \geq \frac{\partial}{\partial x} u_{i}\left(x, \sigma_{n}^{*}(x), b, \lambda, \alpha\right)
$$

\section{Unanimity rule}

In this section we characterize equilibrium offers and payoffs under unanimity rule. In order to do so, we first derive the asymptotic acceptance probabilities. We start by introducing some new notation. Define $x_{\omega}(\lambda)$ as the solution to $\Delta^{\omega}\left(x_{\omega}(\lambda), \underline{\sigma}, \lambda\right)=0$, and write $x_{\omega}(\lambda)=\infty$ if no solution exists. By Assumption $3, x_{H}(\lambda=0) \neq \infty$, and so by continuity there exists $\bar{\lambda}>0$ such that $x_{H}(\lambda) \neq \infty$ for all $\lambda<\bar{\lambda}$. Economically, $x_{\omega}(\lambda)$ is the lowest offer that all voters would accept under unanimity rule if it were somehow revealed that the true state is $\omega$. As such, it is mistake for voters to either accept an offer below $x_{\omega}(\lambda)$ in state $\omega$, or to reject an offer above $x_{\omega}(\lambda)$ in state $\omega$.

Next, define $x_{U}(b, \lambda)$ as the solution to

$$
\Delta^{H}(x, \underline{\sigma}) \ell(\underline{\sigma}) b+\Delta^{L}(x, \underline{\sigma})(1-b)=0 .
$$

Economically, $x_{U}(b, \lambda)$ is the lowest offer that voters accept with probability 1 . For instance, in the opening example $x_{U}(b, \lambda)=3 / 4$. By Assumptions 2, 3 and 5, the lefthand side of (12) is strictly negative at $x=0$, and is strictly increasing in $x$. As such, (12) has at most one solution. If the left hand side is strictly negative at $x=1$, 
define $x_{U}(b, \lambda)=\infty$. Note that if $x_{L}(\lambda) \neq \infty$ then $x_{U}(b, \lambda)<x_{L}(\lambda)$. Consequently acceptance of $x_{U}(b, \lambda)$ in state $L$ is a mistake. Moreover, $x_{U}(b, \lambda)$ is decreasing in $b$.

Define $P_{n}^{\omega}(x, b, \lambda, \alpha)$ as the equilibrium acceptance probability in state $\omega$ given offer $x$. The next result gives the limiting behavior of $P_{n}^{\omega}$ as the number of voters $n$ grows large. The result is an extension of Duggan and Martinelli's (2001) Theorem 4 to cases in which the proposal being voted over is either very unattractive, or very attractive. ${ }^{21}$

Lemma 3 (Limit acceptance probability under unanimity) Suppose unanimity rule is in effect $(\alpha=1)$. Take any $\lambda \in[0, \bar{\lambda})$ and voter belief $b \in(0,1)$. If the offer $x \geq x_{U}(b, \lambda)$ then $P_{n}^{\omega}(x, b, \lambda, 1)=1$ all $n$, for $\omega=L, H$. Otherwise,

$$
\lim _{n \rightarrow \infty} P_{n}^{\omega}(x, b, \lambda, 1)=\left\{\begin{array}{ll}
0 & \text { if } x \leq x_{H}(\lambda) \\
\left(-\frac{\Delta^{H}(x, \underline{\sigma}, \lambda)}{\Delta^{L}(x, \underline{\sigma}, \lambda)} \frac{b}{1-b} \ell(\underline{\sigma})\right)^{\frac{f(\underline{\underline{(\underline{g}} \mid \omega)}}{f(\underline{\sigma} \mid L)-f(\underline{\sigma} \mid H)}} & \text { if } x \in\left(x_{H}(\lambda), x_{U}(b, \lambda)\right)
\end{array} .\right.
$$

The limit acceptance probability $\lim _{n \rightarrow \infty} P^{\omega}(x, b, \lambda, 1)$ is continuous and monotone increasing in both $x$ and $b$.

Lemma 3 reflects the failure of information aggregation under unanimity rule. On the one hand, failure of information aggregation leads offers above $x_{H}$ to be rejected even when $\omega=H$. On the other hand, failure of information aggregation leads offers below $x_{L}$ to be accepted even when $\omega=L$.

Given the limit acceptance probabilities we can characterize the proposer's preferred offers. In order to do so, we must first show that it is legitimate to maximize the proposer's payoff using the limit acceptance probabilities. Mathematically, we must establish that acceptance probabilities converge uniformly:

Lemma 4 (Uniform convergence) For any $\varepsilon>0, P_{n}^{\omega}(\cdot)$ converges uniformly over $[\varepsilon, 1-\varepsilon] \times[\underline{b}, \bar{b}] \times[0,1]$.

\footnotetext{
${ }^{21}$ That is, in Duggan and Martinelli's notation, $\rho$ is either non-positive or exceeds $1 / L$.
} 
The proof of Lemma 4 hinges on the monotonicity of the acceptance probability in the offer $x$. A standard result of real analysis, Helly's Selection Theorem, implies that $P_{n}^{\omega}$ converges uniformly when treated as a function of the offer only, $x$. To establish uniform convergence of $P_{n}^{\omega}$ as a function of $x, b, \lambda$, where $P_{n}^{\omega}$ need not be monotone in $\lambda$, we extend Helly's Selection Theorem. Details are in Lemma A-2, which is stated and proved in the appendix.

When voters use unanimity rule, the vote does not aggregate their information efficiently. Because of this, if it were revealed, the proposer's signal has the capacity to affect the acceptance probability, even when the number of voters is large. Consequently the proposer may try to signal his own information $\sigma_{0}$ with the offer he makes. As is well known, signalling games often possess multiple equilibria. Nonetheless, our next result bounds the proposer's equilibrium offer regardless of the equilibrium played. In doing so, we show that even though information aggregation is imperfect, there is still no equilibrium in which the proposer makes an offer just above the minimum acceptable offer $x_{H}$, and voters accept because they think it signals favorable proposer information.

Proposition 3 (Equilibrium offer under unanimity) Suppose the unanimity rule is in effect $(\alpha=1)$. Then there exists $\check{\lambda}<\bar{\lambda}, \kappa>0$, and $N$ such that for all $\sigma_{0}, \lambda \in[0, \check{\lambda}]$, in any equilibrium the proposer's offer always exceeds $x_{H}(\lambda)+\kappa$ when $n \geq N$, and is always less than $x_{U}(\underline{b}, \lambda)$ (regardless of $n$ ).

Proposition 3 says that the mistakes voters make under unanimity rule force the proposer to offer strictly more than $x_{H}$. Given the uniform convergence of acceptance probabilities established in Lemma 4, it is immediate from Lemma 3 and Proposition 3 that the equilibrium acceptance probability under unanimity rule is bounded uniformly away from zero when the preferences are close to common values.

Corollary 3 (Lower bound on acceptance probability under unanimity) There exists $\check{\lambda}<\bar{\lambda}, \kappa>0$, and $N$ such that for all $\lambda \in[0, \check{\lambda}]$, and $n \geq N$, in any equilibrium the acceptance probability exceeds $\kappa$. 
This Corollary in turn combines with Proposition 2 and Proposition 3 to deliver bounds on the voters' equilibrium payoff under unanimity rule:

Corollary 4 (Bounds on voter payoffs under unanimity) There exists $\check{\lambda}<\bar{\lambda}$, $\kappa>0$, and $N$ such that for all $\lambda \in[0, \check{\lambda}]$, and $n \geq N$, in any equilibrium the payoff of voters exceeds

$$
E_{\sigma_{i}, \omega}\left[\bar{U}^{\omega}\left(\sigma_{i}, \lambda\right)\right]+\kappa
$$

Moreover, if $x_{U}(\underline{b}, \lambda) \neq \infty$, in any equilibrium the payoff of voters is bounded above by

$$
E_{\sigma_{i}, \omega}\left[\bar{U}^{\omega}\left(\sigma_{i}, \lambda\right)\right]+E_{\sigma_{i}, \omega}\left[\Delta^{\omega}\left(x_{U}(\underline{b}, \lambda), \sigma_{i}, \lambda\right)\right]
$$

\section{Majority rule}

We refer to any non-unanimity voting rule $\alpha<1$ as a majority rule. To state our results, we need to generalize the $x_{\omega}(\lambda)$ notation we introduced above. For $\omega=L, H$, define $\sigma_{\omega}(\alpha)$ and $x_{\omega}(\lambda ; \alpha)$ implicitly by

$$
1-F\left(\sigma_{\omega}(\alpha) \mid \omega\right)=\alpha \quad \text { and } \quad \Delta^{\omega}\left(x_{\omega}(\lambda ; \alpha), \sigma_{\omega}(\alpha), \lambda\right)=0
$$

That is, conditional on $\omega$ there is a probability $\alpha$ that the realization of $\sigma_{i}$ exceeds $\sigma_{\omega}(\alpha)$; and $x_{\omega}(\lambda ; \alpha)$ is the proposal that gives a voter $i$ the same payoff as the status quo, given $\omega$ and $\sigma_{i}=\sigma_{\omega}(\alpha)$. As such, if the state $\omega$ were public information, then an offer just above $x_{\omega}(\lambda ; \alpha)$ would be accepted with probability converging to 1 as the number of voters $n$ grows large. Note that $x_{\omega}(\cdot ; \alpha=1) \equiv x_{\omega}(\cdot)$, so this notation contains the notation of the prior section as a special case. Moreover, under pure common values $(\lambda=0)$ the value $x_{\omega}(\lambda ; \alpha)$ is independent of the voting rule $\alpha$.

The existing strategic voting literature has established that majority rule perfectly aggregates information in the limit (i.e., as the number of voters grows large). In terms of the above notation, asymptotically perfect information aggregation means that the limiting acceptance probability given state $\omega$ is zero if the offer $x$ is less than $x_{\omega}(\lambda ; \alpha)$, 
and is one if the offer $x$ exceeds $x_{\omega}(\lambda ; \alpha)$. As such, the limiting acceptance probability is discontinuous. In contrast, the acceptance probability for any finite number of voters $n$ is continuous (see Corollary 1). An important consequence of these observations is that when majority rule is used the acceptance probabilities do not converge uniformly to their limit - in sharp contrast to the case of unanimity rule (Lemma 4).

Because of this failure of uniform convergence, it is not possible to first analyze the equilibrium of the limit game, and then to show that it is also the limit of equilibria of finite games. We deal with this complication by first extending the existing strategic voting literature to the case where the proposal being voted over varies with the number of voters. Since there is no reason to require the proposer's offers to have a well-defined limit, we state our result in terms of the limits infimum and supremum. We show that, as in Feddersen and Pesendorfer (1997) and Duggan and Martinelli (2001), the aggregate response of the voting group to an offer $x$ matches that which would be obtained under full information.

\section{Lemma 5 (Acceptance probabilities under majority) Suppose a majority voting} rule $\alpha<1$ is in effect. Take any $\lambda \in[0,1]$, and consider a sequence of offers $x_{n}$. If $\liminf x_{n}>x_{\omega}(\lambda ; \alpha)$ then $P_{n}^{\omega}\left(x_{n}\right) \rightarrow 1$. If $\lim \sup x_{n}<x_{\omega}(\lambda ; \alpha)$ then $P_{n}^{\omega}\left(x_{n}\right) \rightarrow 0$.

We next use Lemma 5 to characterize the proposer's equilibrium offer. First, note that the acceptance probabilities are asymptotically constant over each of the ranges $\left[0, x_{H}\right),\left(x_{H}, x_{L}\right)$, and $\left(x_{L}, 1\right]$. Consequently, when facing a large number of voters using majority rule, the proposer will never make an offer that lies far from the lower ends of these ranges, i.e., $0, x_{H}, x_{L}$. Since the offer 0 is always rejected when the number of voters is large, and the proposer prefers agreement to disagreement, the proposer's choice approximately boils down to $x_{H}$ versus $x_{L}$. To determine the proposer's choice between the two, for any $\sigma_{0}$ define

$$
W\left(\sigma_{0} ; \lambda, \alpha\right) \equiv p^{H}\left(\sigma_{0}\right) V^{H}\left(x_{H}, \sigma_{0}\right)+p^{L}\left(\sigma_{0}\right) \bar{V}^{L}\left(\sigma_{0}\right)-E\left[V^{\omega}\left(x_{L}, \sigma_{0}\right) \mid \sigma_{0}\right] .
$$


The function $W$ has the following interpretation: the first two terms are the proposer's expected payoff from offering $x_{H}$ if this offer is accepted when $\omega=H$ and rejected when $\omega=L$. The final term is the proposer's expected payoff from offering $x_{L}$ if this offer is always accepted. In Lemma 5 we established that approximately this acceptance behavior is obtained as the number of voters grows large. As we show formally in Lemma 6 below, it follows that a proposer facing a large number of voters will offer $x_{H}$ whenever $W\left(\sigma_{0} ; \lambda, \alpha\right)>0$; and will offer $x_{L}$ whenever $W\left(\sigma_{0} ; \lambda, \alpha\right)<0$.

It is easily verified that if $\sigma_{0}$ affects only the proposer's information, and not his preferences (i.e., $V^{\omega}$ and $\bar{V}^{\omega}$ are independent of $\sigma_{0}$ ), then $W$ is strictly increasing in $\sigma_{0}$. That is, when the proposer is more confident that the true state is $H$ he is more likely to make the minimum offer acceptable in that state, $x_{H}$. Formally, there exists a cutoff $\hat{\sigma}_{0}$ such that $W$ is positive if and only if $\sigma_{0}>\hat{\sigma}_{0}$. By continuity, the same is true whenever $\sigma_{0}$ does not affect the proposer's preferences too strongly.

Next, consider what economic circumstances lead $W$ to be positive, and so to the proposer making the offer $x_{H}$ against majority rule. First, $W$ is increasing in $x_{L}$ and decreasing in $x_{H}$. As such, $W$ is more likely to be positive if a voter's payoff relative to the status quo in state $L$ is low (i.e., $\Delta^{L}$ low); or a voter's payoff relative to the status quo in state $H$ is high (i.e., $\Delta^{H}$ high). Second, turning to the proposer's own preferences, $W$ is more likely to be positive if his status quo payoff in state $L$ (i.e., $\bar{V}^{L}$ ) is high; or the cost of increasing the offer in state $H$ (i.e., $\left|\frac{\partial V^{H}}{\partial x}\right|$ ) is high; or the value of having an offer accepted in state $L$ (i.e., $V^{L}$ ) is low.

We now turn to our formal result characterizing equilibrium offers against majority rule. Note that because under majority voting voters' signals asymptotically reveal the true realization of $\omega$, there is no scope for the proposer's offer to convey useful information. Consequently the signalling aspect of the bargaining game disappears. The equilibrium outcome is then asymptotically unique. ${ }^{22}$

\footnotetext{
${ }^{22}$ More accurately, the equilibrium is unique within the class of symmetric voter equilibria, and given our standard equilibrium selection rule that chooses a responsive equilibrium whenever one exists.
} 
It follows that when voters hold a majority vote we can precisely characterize the expected equilibrium payoffs of both the proposer and voters. Doing so, however, requires handling one further technical issue. We must show that as the number of voters grows large, equilibrium offers and acceptance probabilities converge uniformly with respect to the proposer's signal $\sigma_{0}$. Our next result shows this is indeed true, even though (as discussed above) the acceptance probability function does not converge uniformly.

Lemma 6 (Equilibrium offer under majority) Suppose a majority voting rule $\alpha<$ 1 is in effect. Then:

(1) If $x_{L}(\lambda ; \alpha) \neq \infty \neq x_{H}(\lambda ; \alpha)$, then for any $\varepsilon, \delta>0$ there exists $N(\varepsilon, \delta)$ such that

(a) If $W\left(\sigma_{0}\right)>\varepsilon$ and $n \geq N(\varepsilon, \delta)$ then for any equilibrium offer $x,\left|x-x_{H}(\lambda ; \alpha)\right|<$ $\delta ; P_{n}^{H}\left(x \mid \sigma_{0}\right)>1-\delta$; and $P_{n}^{L}\left(x \mid \sigma_{0}\right)<\delta$.

(b) If $W\left(\sigma_{0}\right)<-\varepsilon$ and $n \geq N(\varepsilon, \delta)$ then for any equilibrium offer $x,\left|x-x_{L}(\lambda, \alpha)\right|<$ $\delta ; P_{n}^{H}\left(x \mid \sigma_{0}\right)>1-\delta ;$ and $P_{n}^{L}\left(x \mid \sigma_{0}\right)>1-\delta$.

(2) If $x_{H}(\lambda ; \alpha) \neq \infty$ and $x_{L}(\lambda ; \alpha)=\infty$ then for any $\delta>0$ there exists $N(\delta)$ such that for any equilibrium offer $x,\left|x-x_{H}(\lambda ; \alpha)\right|<\delta$ and $P_{n}^{H}\left(\cdot \mid \sigma_{0}\right)>1-\delta$ for all $\sigma_{0}$ when $n \geq N(\delta)$.

(3) If $x_{L}(\lambda ; \alpha)=x_{H}(\lambda ; \alpha)=\infty$, for any $\delta>0$ there exists $N(\delta)$ such that for any equilibrium offer $x, P_{n}^{\omega}\left(x \mid \sigma_{0}\right)<\delta$ for all $\sigma_{0}, \omega=L, H$ when $n \geq N(\delta)$.

For use below, we set $W\left(\sigma_{0} ; \lambda, \alpha\right)=\infty$ when $x_{H}(\lambda ; \alpha) \neq \infty$ and $x_{L}(\lambda ; \alpha)=\infty$. Lemma 6 says that the proposer will make an offer close to $x_{H}(\lambda ; \alpha)$ (respectively, $\left.x_{L}(\lambda ; \alpha)>x_{H}(\lambda ; \alpha)\right)$ after observing a $\sigma_{0}$ such that $W\left(\sigma_{0}\right)$ is strictly positive (negative). As stated, it does not cover equilibrium behavior in the knife-edge case that $W\left(\sigma_{0}\right)=0$. From above, however, we know that whenever the effect of $\sigma_{0}$ on the proposer's preferences is weak enough, $W$ equals zero for at most one value of $\sigma_{0}$. More generally, for the remainder of the paper we make the following very mild assumption:

Assumption $6 W\left(\sigma_{0} ; \lambda, \alpha\right)=0$ for at most finitely many values of $\sigma_{0}$ when $x_{L}(\lambda ; \alpha) \neq$ $\infty \neq x_{H}(\lambda ; \alpha)$. 
From Lemma 6 it is straightforward to establish the limiting expected payoffs of the proposer and the voters under any majority voting rule. Notationally, we write $\Pi_{n}^{* P}(\lambda, \alpha)$ and $\Pi_{n}^{* V}(\lambda, \alpha)$ for the proposer's and voters' expected equilibrium payoffs. Immediate from Lemma 6, we have:

Proposition 4 (Equilibrium payoffs under majority) Suppose a majority voting rule $\alpha<1$ is in effect and $x_{H}(\lambda ; \alpha) \neq \infty$. Then the equilibrium payoffs satisfy:

$$
\begin{aligned}
\Pi_{n}^{* V}(\lambda, \alpha) \rightarrow & E_{\sigma_{i}, \omega}\left[\bar{U}^{\omega}\left(\sigma_{i}\right)\right]+\int_{\sigma_{0} \text { s.t. } W\left(\sigma_{0}\right)<0} E_{\sigma_{i}, \omega}\left[\Delta^{\omega}\left(x_{L}, \sigma_{i}, \lambda\right) \mid \sigma_{0}\right] d F_{0}\left(\sigma_{0}\right) \\
& +\int_{\sigma_{0} \text { s.t. } W\left(\sigma_{0}\right)>0} p^{H}\left(\sigma_{0}\right) E_{\sigma_{i}}\left[\Delta^{H}\left(x_{H}, \sigma_{i}, \lambda\right) \mid H\right] d F_{0}\left(\sigma_{0}\right) \\
\Pi_{n}^{* P}(\lambda, \alpha) \rightarrow & \int_{\sigma_{0} \text { s.t. } W\left(\sigma_{0}\right)<0} E_{\omega}\left[V^{\omega}\left(x_{L}, \sigma_{0}\right) \mid \sigma_{0}\right] d F_{0}\left(\sigma_{0}\right) \\
& +\int_{\sigma_{0} \text { s.t. } W\left(\sigma_{0}\right)>0}\left(p^{H}\left(\sigma_{0}\right) V^{H}\left(x_{H}, \sigma_{0}\right)+p^{L}\left(\sigma_{0}\right) \bar{V}^{L}\left(\sigma_{0}\right)\right) d F_{0}\left(\sigma_{0}\right) .
\end{aligned}
$$

From the preceding results, one can see that although majority rule aggregates information efficiently independently of whether or not the proposer observes an informative signal, the proposer's signal does affect the offer he makes. A priori, one might conjecture that since voters and the proposer have opposing preferences, voters would prefer to deal with an uninformed proposer. However, there are circumstances under which this is not true. Specifically, consider the case in which proposer preferences are independent of $\sigma_{0}$, and the cutoff $\hat{\sigma}_{0}$ at which $W=0$ is low. Here, the proposer makes the high offer $x_{L}$ whenever $\sigma_{0}<\hat{\sigma}_{0}$, and the low offer $x_{H}$ otherwise. In contrast, consider the offer made by a completely uninformed proposer with the same preferences. The $W$ function for this proposer is simply the integral of $W\left(\sigma_{0}\right)$ over all possible realizations of the informed proposer's signal $\sigma_{0}$. Because $\hat{\sigma}_{0}$ was assumed to be low, this integral is positive, and so the uninformed proposer makes the low offer $x_{H}$. From Proposition 2, it follows that voters prefer to deal with the uninformed proposer whenever they have close to common values preferences, since the informed proposer makes the high offer $x_{L}$ at least sometimes. 


\section{Comparing majority and unanimity voting rules}

We are now ready to compare the equilibrium payoffs of the proposer and voters under majority rule to those under unanimity rule. We focus on cases in which voter preferences are not too far from pure common values (i.e., $\lambda$ close enough to 0 ). As we will see, the comparison depends critically on the sign of the $W$ function, which is in turn affected by both the proposer's and responders' preferences (see discussion on page 25). One particularly transparent determinant of $W^{\prime}$ 's sign is the proposer's status quo payoff in state $L$ (i.e., $\bar{V}^{L}$ ). We often refer back to this case, and refer to the proposer as being more (respectively, less) pro-agreement when $\bar{V}^{L}$ is low (high).

VOTER PREFERENCES OVER THE VOTING RULE

First, suppose $W(\cdot ; \lambda, \alpha)>0$ (e.g., the proposer is less pro-agreement). Directly from Proposition 4, for any majority voting rule $\alpha<1$,

$$
\Pi_{n}^{* V}(\lambda, \alpha) \rightarrow E_{\sigma_{i}, \omega}\left[\bar{U}^{\omega}\left(\sigma_{i}, \lambda\right)\right]+p^{H} E_{\sigma_{i}}\left[\Delta^{H}\left(x_{H}(\lambda ; \alpha), \sigma_{i}, \lambda\right) \mid H\right]
$$

The first term is the voters' payoff under the status quo. In general, the second term can be positive or negative. However, by definition, $\Delta^{H}\left(x_{H}(\lambda ; \alpha), \sigma_{H}, \lambda\right)=0$, and $\Delta^{H}$ is independent of $\sigma_{i}$ when $\lambda=0$. Consequently $E_{\sigma_{i}}\left[\Delta^{H}\left(x_{H}(\lambda ; \alpha) ; \sigma_{i}, \lambda\right) \mid H\right]$ approaches 0 as $\lambda \rightarrow 0$, and so the voters' payoff approaches their status quo payoff. That is, against majority rule the proposer is able to reduce the voters' payoff all the way to their outside option.

From Corollary 4, the voters' equilibrium payoff when they use unanimity rule is bounded away from their status quo payoff $E_{\sigma_{i}, \omega}\left[\bar{U}^{\omega}\left(\sigma_{i}, \lambda\right)\right]$. It is then immediate that voters are better off under unanimity rule when $W(\cdot ; \lambda=0, \alpha)>0$.

Proposition 5 (Voters better off under unanimity) Fix a majority rule $\alpha<1$, and suppose that $W(\cdot ; \lambda=0, \alpha)>0$. Then there exists $\check{\lambda}>0$ such that whenever $\lambda<\check{\lambda}$, voters strictly prefer unanimity rule to the majority rule $\alpha$ (regardless of the equilibrium played). 
Next, suppose instead that $W(\cdot ; \lambda, \alpha)<0$ (e.g., the proposer is more pro-agreement). In this case, the proposer's offer to voters using majority rule converges to $x_{L}(\lambda ; \alpha)$, the offer which is required to guarantee acceptance in both state $L$ and $H$. By definition, $x_{L}(\lambda ; \alpha) \leq 1$ when $W(\cdot ; \lambda, \alpha)<0$. Moreover, close to common values $x_{L}(\lambda ; \alpha)$ is approximately constant in $\alpha$, and so $x_{U}(\underline{b}, \lambda)<x_{L}(\lambda ; \alpha)$. When voters use unanimity rule the proposer offers no more than $x_{U}(\underline{b}, \lambda)$, since this offer guarantees acceptance (Lemma 3). Since voters prefer higher offers (Proposition 2) it follows that:

Proposition 6 (Voters better off under majority) Fix a majority voting rule $\alpha<$ 1 , and suppose that $W(\cdot ; \lambda=0, \alpha)<0$. Then there exists $\check{\lambda}>0$ such that whenever $\lambda \leq \check{\lambda}$, for all $n$ large enough voters strictly prefer the majority rule $\alpha$ to unanimity rule (regardless of the equilibrium played).

To illustrate Propositions 5 and 6, consider gradually increasing the proposer's status quo payoff $\bar{V}^{L}$ in state $L$. When this is low, the proposer is anxious to obtain agreement, and so makes the high offer $x_{L}$ against majority rule. Against unanimity rule he can obtain agreement more cheaply (by offering $x_{U}$, for example), and so voters prefer majority rule (Proposition 6). In this case, voters' mistakes under unanimity hurt them. As the proposer's status quo payoff rises, reaching agreement at any cost becomes less important to him, and under majority rule he reduces his offer to $x_{H}<x_{L}$. However, he cannot reduce his offer against unanimity rule to $x_{H}$, since then he would be rejected all the time. Now, voters prefer unanimity rule (Proposition 5), since their mistakes under unanimity actually help them.

\section{Proposer PREFERENCES OVER THE VOtING RULE}

We now turn to proposer preferences. In two significant cases, proposer preferences over voting rules are diametrically opposed to voters' preferences.

First, suppose $W(\cdot ; \lambda, \alpha)<0$ (e.g., the proposer is more pro-agreement). In this case the proposer offers $x_{L}$ to voters using majority rule and the offer is always accepted. 
On the other hand, if voters use unanimity rule, the proposer is able to obtain certain acceptance with a lower offer. As such, the proposer prefers to face voters using unanimity rule. This result (Proposition 7, below) combines with Proposition 6 to show that when $W(\cdot ; \lambda, \alpha)<0$ voters and the proposer have opposite preferences over the voting rule.

Proposition 7 (Proposer better off under unanimity) Fix a majority voting rule $\alpha<1$, and suppose that $W(\cdot ; \lambda=0, \alpha)<0$. Then there exists $\check{\lambda}>0$ such that whenever $\lambda \leq \check{\lambda}$, for all $n$ large enough the proposer strictly prefers unanimity rule to the majority rule $\alpha$ (regardless of the equilibrium played).

Second, suppose $W(\cdot ; \lambda, \alpha)>0$ (e.g., the proposer is less pro-agreement). In this case, under majority rule the proposer makes the low offer $x_{H}$. It follows that, compared to unanimity rule, majority rule results in more agreement in state $H$ and less agreement in state $L$. As such, if agreement in state $L$ is socially inefficient, then total surplus in that state is higher under majority rule than under unanimity rule. Since voters are better off under unanimity rule (Proposition 5), it loosely follows that the proposer is correspondingly worse off under unanimity rule. However, making this argument precise requires a further assumption to rule out any gains from reallocating resources from one state to another. Specifically:

Assumption $7 U^{\omega}\left(x, \sigma_{i}, \lambda\right)$ and $V^{\omega}\left(x, \sigma_{0}\right)$ are linear in $x$, and $U_{x}^{\omega}\left(x, \sigma_{i}, \lambda\right) / V_{x}^{\omega}\left(x, \sigma_{0}\right)$ is independent of $\omega, \sigma_{i}$ and $\sigma_{0}$.

Assumption 7 is satisfied in many standard environments. (We also stress that although it is sufficient for the results that follow, it is not necessary.) It implies the existence of a constant $C>0$ such that $U^{\omega}\left(x, \sigma_{i}, \lambda\right)+C V^{\omega}\left(x, \sigma_{0}\right)$ is independent of $x$, for all $\omega, \sigma_{0}, \sigma_{i}$, and $\lambda$. In other words, agreement creates the same total surplus independent of the offer $x$ - which affects only the division of surplus between the bargaining parties. Agreement in state $L$ is socially inefficient if

$$
U^{L}\left(\cdot, \sigma_{i}, \lambda\right)+C V^{L}\left(\cdot, \sigma_{0}\right)<\bar{U}^{L}\left(\sigma_{i}, \lambda\right)+C \bar{V}^{L}\left(\sigma_{0}\right)
$$


Note that (15) always holds when fully-informed voters would reject even the offer $x=1$ in state $L$ (i.e., $\left.x_{L}(\lambda ; \alpha)=\infty\right)$ and the proposer is close to indifferent between the offer $x=1$ and the status quo (i.e., $V^{L}\left(1, \sigma_{0}\right)$ is sufficiently close to $\left.\bar{V}^{L}\left(\sigma_{0}\right)\right)$. Note also that by Assumptions 3 and 4,

$$
U^{H}\left(\cdot, \sigma_{i}, \lambda\right)+C V^{H}\left(\cdot, \sigma_{0}\right)>\bar{U}^{H}\left(\sigma_{i}, \lambda\right)+C \bar{V}^{H}\left(\sigma_{0}\right)
$$

so that agreement always creates surplus in state $H$.

We are now ready to establish our formal result. When $W(\cdot ; \lambda, \alpha)>0$ we know that the proposer offers $x_{H}(\lambda ; \alpha)$ to voters using majority rule, and the voters accept if and only if the state is $H$. So if agreement in state $L$ is inefficient (i.e., if (15) holds), majority rule maximizes total surplus. In contrast, if voters use unanimity rule, the proposer offers strictly more than $x_{H}(\lambda ; \alpha)$, and his offer is accepted with strictly positive probability in state $L$. Since total surplus is lower and voters strictly prefer unanimity to majority, it follows that the proposer has exactly the opposite preferences.

Proposition 8 (Proposer prefers majority) Suppose that Assumption 7 and inequality (15) hold for $\lambda=0$. Fix a majority voting rule $\alpha<1$. If $W(\cdot ; \lambda, \alpha)>0$ then there exists $\check{\lambda}>0$ such that whenever $\lambda \leq \check{\lambda}$, for all $n$ large enough the proposer strictly prefers the majority rule $\alpha$ to unanimity rule (regardless of the equilibrium played).

\section{PARETO DOMINANCE OF UNANIMITY RULE}

Propositions 7 and 8 give conditions under which the proposer and voters have opposite preferences over the voting rule used by the voters. However, and as we saw in the opening example, there are also cases in which both sides strictly prefer unanimity rule to majority rule. The two key requirements for this to occur are that (i) agreement creates social surplus in state $L$ as well as state $H$, and (ii) the proposer offers $x_{H}$ to voters using majority rule. Under these conditions, total surplus may be higher under unanimity rule, since under majority rule voters reject the offer $x_{H}$ in state $L$. For instance, in the opening example both sides prefer unanimity when the debtor's outside option $\bar{V}$ 
lies between 20 and 95: whenever $\bar{V}>20$ the debtor offers $x_{H}=1 / 2$ to creditors using majority rule, and whenever $\bar{V}<95$ agreement in state $L$ generates a social surplus of at least $120-95=25$. We now establish this result more generally.

We first consider the case in which the proposer's signal is completely uninformative, and voters' preferences are pure common values $(\lambda=0)$. For this case, we establish:

\section{Proposition 9 (Pareto dominance of unanimity with uninformed proposer)} Suppose $\lambda=0$ and the proposer's signal is completely uninformative. There exist preferences (i.e., $\left.U^{\omega}, \bar{U}^{\omega}, V^{\omega}, \bar{V}^{\omega}\right)$ such that for all $n$ sufficiently large both the proposer and voters strictly prefer unanimity rule (regardless of the equilibrium played). In contrast, there do not exist preferences under which the proposer and voters both prefer majority rule for all $n .^{23}$

We prove this result in reverse order, and first establish that there are no preferences such that both sides prefer majority rule. When the proposer's signal is uninformative, the function $W$ is a constant independent of $\sigma_{0}$. A necessary condition for voters to prefer majority rule to unanimity rule is $W \leq 0$ : for if instead $W>0$, from Proposition 5 voters strictly prefer unanimity rule. Since $W \leq 0$, the proposer at least weakly prefers having $x_{L}$ accepted always to having $x_{H}$ accepted only in state $H$. He clearly strictly prefers having $x_{U}<x_{L}$ accepted always, which is possible under unanimity rule, to having $x_{L}$ accepted always. So for all $n$ large enough, the proposer strictly prefers unanimity rule when $W \leq 0$.

We now establish that for some preferences both sides prefer unanimity rule. Choose preferences such that $W=0$ (in the opening example, this is the point $\bar{V}=20$ ). Under these preferences, the proposer strictly prefers unanimity rule to majority rule for $n$ large enough, by the same argument as above. By continuity, the same is true if $\bar{V}^{L}$ is increased slightly, so that $W>0$. Under these perturbed preferences, the voters also prefer unanimity whenever $n$ is sufficiently large.

\footnotetext{
${ }^{23}$ Note that Proposition 9 does not require Assumption 7 to hold.
} 
(Note that when $\lambda=0, W=0$ certainly implies that agreement in state $L$ Pareto dominates the status quo, i.e., is socially efficient. To see this, recall that $W=0$ says that the proposer is indifferent between the offer $x_{H}$ being accepted in state $H$ only, and the more costly offer $x_{L}$ being accepted in both states. If the proposer weakly preferred the status quo to outcome $x_{L}$ in state $L$, this indifference condition would not hold. So the proposer strictly prefers outcome $x_{L}$ to the status quo in state $L$, while by construction voters are indifferent between the two.)

The intuition behind Proposition 9 is as follows. Voters make mistakes under unanimity rule, and these mistakes lead the proposer to make a higher offer under unanimity rule. This higher offer in turn increases the acceptance probability in state $L$. Because agreement is socially efficient in state $L$, overall social surplus is thereby increased. In contrast, under majority rule the offer that is required to attain the same acceptance probability (and thus social surplus) in state $L$ is too expensive for the proposer.

Proposition 9 establishes that there are conditions under which unanimity Pareto dominates majority voting. As stated, it covers only the case in which the proposer's signal is completely uninformative and the voters have pure common values. Although these assumptions greatly simplify the proof, they are not essential. More generally, we can establish the following:

\section{Proposition 10 (Pareto dominance of unanimity with informed proposer)} Suppose that Assumption 7 holds, and that voter preferences are such that $x_{L}(\lambda=0)<1$. Then provided voter information is sufficiently poor $(\ell(\underline{\sigma})$ close enough to 1$)$ there exist proposer preferences such that for any majority rule $\alpha<1$, there exist $N, \check{\lambda}>0$ such that both the proposer and voters strictly prefer unanimity to the majority rule $\alpha$ for $n \geq N$ and $\lambda \leq \check{\lambda}$.

The proof of this last result is conceptually similar to that of Proposition 9, but is considerably more involved. It is omitted for space reasons, and is available from a technical appendix posted on the authors' webpages. 


\section{Concluding remarks}

In this paper we have analyzed a strategic voting game in which the agenda is set endogenously. We have shown that in such an environment, unanimity rule may be the preferred voting rule not only of the voting group, but also of the opposing party as well. These results contrast sharply with the results of the existing strategic voting literature that has analyzed voting over exogenous agendas.

Inevitably our analysis has neglected some important issues. We focus almost exclusively on equilibrium payoffs as the group size grows large. The chief reason for this focus is that it allows us to establish our results with fewer assumptions on preferences and the distributional properties of agents' information. Numerical simulations suggest that the group size needed for our asymptotic results to apply is not large - in many cases the equilibrium with ten agents is very close to the limiting equilibrium.

Our analysis has focused primarily on common values environments in which voters' preferences are aligned. Of course, when preferences are close to pure private values agreement is very hard to obtain under unanimity rule. Related, to ensure that our results do not depend on complete preference alignment, we have established all our main results for the case in which voter preferences are not perfectly aligned, but instead are "sufficiently close" to common values. An alternative robustness check would be to consider the case in which a fraction $1-\varepsilon$ voters have pure common values preferences, while the remaining fraction $\varepsilon$ have extreme private valuations. In such circumstances, unanimous agreement would be impossible to obtain asymptotically. However, a version of our results should still hold when the number of voters is not too large. As we discussed above, acceptance probabilities converge relatively quickly to their limiting expressions.

We conclude with a discussion of implications our analysis has for pre-vote communication, i.e., deliberation. In our analysis, the role of voting is to aggregate information, and no communication is permitted. As is well-known, when voters have biases, full information sharing during communication is not always possible (see Coughlan 2000, 
Meirowitz 2005, Austen-Smith and Feddersen, 2006). ${ }^{24}$ In contrast, when there are no biases, as in the pure common values case, voters would share their information truthfully when voting is over an exogenous agenda. The same is true when voting is over an endogenous agenda and voters are worse off under unanimity rule due to mistakes. Note however that the mistakes often benefit voters by inducing the proposer to make a better offer. In this case, voters would want to ex ante commit not to communicate ex post (i.e., after the offer is made). ${ }^{25}$ Of course, ex post they still wish to change their minds and communicate, but when the number of voters is large such communication will be hard to achieve without pre-existing arrangements. As such, our analysis complements Austen-Smith and Feddersen's (2006) result that when the agenda is exogenous voters may not communicate truthfully under unanimity rule when their interests are imperfectly aligned. Our analysis implies that even when interests are perfectly aligned, voters may still not communicate truthfully, because by refraining from communication they generate a better (endogenous) offer.

\section{References}

David Austen-Smith and Jeffrey S. Banks. Information aggregation, rationality, and the condorcet jury theorem. American Political Science Review, 90(1):34-45, March 1996.

David Austen-Smith and Timothy Feddersen. Delibration, preference uncertainty, and voting rules. American Political Science Review, 100(2):209-217, May 2006.

D. P. Baron and J. A. Ferejohn. Bargaining in legislatures. American Political Science Review, 83:1181-1206, 1989.

\footnotetext{
${ }^{24}$ See also Doraszelski et al (2003), and Gerardi and Yariv (forthcoming) for communication in strategic voting games. See also Caillaud and Tirole (2006) which adopts a mechanism design approach to communication, and emphasizes the need to distill information selectively to create persuasion cascades.

${ }^{25}$ An additional reason why voters may not communicate truthfully is that the proposer may be able to observe their messages. In this case, voters have an incentive to convince the proposer that the state is $L$, and that, as such, he needs to make an attractive offer.
} 
Bernard Caillaud and Jean Tirole. Consensus building: How to persuade a group. Working Paper, 2006.

D. Cardona and C. Ponsati. Bargaining one-dimensional social choices. Working Paper, 2005.

Suchan Chae and Hervé Moulin. Bargaining among groups: an axiomatic viewpoint. Working Paper, 2004.

Kalyan Chatterjee and Larry Samuelson. Bargaining with two-sided incomplete information: An infinite horizon model with alternating offers. Review of Economic Studies, 54(2):175-192, April 1987.

S. Cho and J. Duggan. Uniqueness of stationary equilibria in a one-dimensional model of bargaining. Journal of Economic Theory, 113:118-130, 2003.

Peter J. Coughlan. In defense of unanimous jury verdicts: Mistrials, communication, and strategic voting. American Political Science Review, 94(2):375-393, June 2000.

Raymond Deneckere and Meng-Yu Liang. Bargaining with interdependent values. Econometrica, 74(2):1309-1364, 2006.

Ulrich Doraszelski, Dino Gerardi, and Francesco Squintani. Communication and voting with double-sided information. Contributions to Theoretical Economics, 3(1), 2003.

John Duggan and César Martinelli. A bayesian model of voting in juries. Games and Economic Behavior, 37(2):259-294, November 2001.

Alexander Elbittar, Andrei Gomberg, and Laura Sour. Group decision making in ultimatum bargaining: A laboratory study. Working Paper, 2004.

Robert Evans. Sequential bargaining with correlated values. Review of Economic Studies, 56:499-510, 1989. 
Timothy Feddersen and Wolfgang Pesendorfer. The swing voter's curse. American Economic Review, 86(3):408-424, June 1996.

Timothy Feddersen and Wolfgang Pesendorfer. Voting behavior and information aggregation in elections with private information. Econometrica, 65(5):1029-1058, September 1997.

Timothy Feddersen and Wolfgang Pesendorfer. Convicting the innocent: The inferiority of unanimous jury verdicts under strategic voting. American Political Science Review, 92(1):23-35, March 1998.

Dino Gerardi and Leeat Yariv. Deliberative voting. Journal of Economic Theory, forthcoming.

Emeric Henry. Aggregation of information and supermajorities. Working Paper, 2006.

John Kennan and Robert Wilson. Bargaining with private information. Journal of Economic Literature, 31(1):45-104, March 1993.

Alejandro M Manelli. Cheap talk and sequential equilibria in signaling games. Econometrica, 64(4):917-942, July 1996.

Paola Manzini and Marco Mariotti. Alliances and negotiations. Journal of Economic Theory, 121(1):128-141, March 2005.

César Martinelli. Would rational voters acquire costly information? Journal of Economic Theory, 2005.

Andrew McLennan. Consequences of the condorcet jury theorem for beneficial information aggregation by rational agents. The American Political Science Review, 92(2): 413-418, June 1998.

Adam Meirowitz. Designing institutions to aggregate preferences and information. Working paper, 2005. 
Nicola Persico. Committee design with endogenous information. Review of Economic Studies, 71(1):165-191, January 2004.

William Samuelson. Bargaining under asymmetric information. Econometrica, 52(4): 995-1006, July 1984.

Urs Schweizer. Litigation and settlement under two-sided incomplete information. Review of Economic Studies, 56:163-178, 1989.

Daniel R Vincent. Bargaining with common values. Journal of Economic Theory, 48: 47-62, 1989.

Leeat Yariv. When majority rule yields majority ruin. Working Paper, 2004.

\section{Appendix A}

We repeatedly use the following straightforward result. The proof is available on request.

Lemma A-1 $F(\sigma \mid H) / F(\sigma \mid L)$ is increasing in $\sigma$, and is bounded above by 1 . Consequently, $F(\sigma \mid H) \leq F(\sigma \mid L)$, and is strict if $\sigma \in(\underline{\sigma}, \bar{\sigma})$. Moreover, $(1-F(\sigma \mid H)) /(1-F(\sigma \mid L))$ is increasing in $\sigma$, and is bounded above by $\ell(\bar{\sigma})>1$.

Proof of Lemma 1: First note that if $Z(x, \sigma)=0$, then it must be the case that $\Delta^{H}(x, \sigma)>0$ by Assumption 2. This implies that $Z(x, \sigma)$ is strictly increasing in $\sigma$ whenever $Z(x, \sigma) \geq 0$. In turn, $Z\left(x, \sigma^{\prime}\right)<0$ for all $\sigma^{\prime}<\sigma$ if $Z(x, \sigma)=0$.

Part 1: By definition, if $x<\underline{x}_{n}$ then $Z(x, \cdot)<0$, while if $x>\bar{x}_{n}$ then $Z(x, \cdot)>0$. For $x \in\left[\underline{x}_{n}, \bar{x}_{n}\right]$ we claim that $Z(x, \sigma)=0$ for some unique $\sigma$, which we write as $\sigma^{*}(x)$. Existence is immediate, since $\max _{\sigma} Z(x, \sigma) \geq 0 \geq \min _{\sigma} Z(x, \sigma)$, and $Z(x, \sigma)$ is continuous in $\sigma$. Uniqueness follows from the result we have just shown that $Z(x, \sigma)$ is strictly increasing in $\sigma$ whenever $Z(x, \sigma) \geq 0$.

Part 2: To see that $\sigma^{*}(x)$ is decreasing, consider $x$ and $x^{\prime}>x$ in $\left(\underline{x}_{n}, \bar{x}_{n}\right)$. Since $Z\left(x, \sigma^{*}(x)\right)=0$, it follows that $Z\left(x^{\prime}, \sigma^{*}(x)\right)>0$. Since $Z\left(x^{\prime}, \sigma\right)$ is increasing in $\sigma$ 
it must be the case that $\sigma^{*}\left(x^{\prime}\right)<\sigma^{*}(x)$. By the Implicit Function Theorem, $\sigma(x)$ is continuously differentiable over $\left(\underline{x}_{n}, \bar{x}_{n}\right)$. To see $\sigma^{*}\left(\underline{x}_{n}\right)=\bar{\sigma}$, suppose to the contrary that $\sigma^{*}\left(\underline{x}_{n}\right)<\bar{\sigma}$. By definition $Z\left(\underline{x}_{n}, \sigma^{*}\left(\underline{x}_{n}\right)\right)=0$, and so $Z\left(\underline{x}_{n}, \bar{\sigma}\right)>0$. By continuity there exists an $x<\underline{x}_{n}$ such that $Z(x, \bar{\sigma})>0$ as well. This contradicts the definition of $\underline{x}_{n}$. Likewise, to see $\sigma^{*}\left(\bar{x}_{n}\right)=\underline{\sigma}$ suppose to the contrary that $\sigma^{*}\left(\bar{x}_{n}\right)>\underline{\sigma}$. By definition $Z\left(\bar{x}_{n}, \sigma^{*}\left(\bar{x}_{n}\right)\right)=0$ which implies that $Z\left(\bar{x}_{n}, \underline{\sigma}\right)<0$. By continuity there exists an $x$ such that $x>\bar{x}_{n}$ and $Z(x, \sigma)<0$, contradicting the definition of $\bar{x}_{n}$.

Part 3: Immediate from the observation that as $n \rightarrow \infty$,

$$
\ell(\sigma)\left(\frac{F(\sigma \mid H)}{F(\sigma \mid L)}\right)^{n-n \alpha}\left(\frac{1-F(\sigma \mid H)}{1-F(\sigma \mid L)}\right)^{n \alpha-1}
$$

converges to 0 and $\infty$ respectively for $\sigma=\underline{\sigma}, \bar{\sigma}$.

Proof of Lemma 2: Define $b_{\varphi}=\int p^{H}\left(\sigma_{0}\right) \varphi\left(d \sigma_{0}\right)$. The equilibrium $\sigma_{n}^{*}$ of the voting stage of the game is the unique solution to $Z\left(x, \sigma, b_{\varphi}\right)=0$, provided a solution exists; is $\underline{\sigma}$ if $Z\left(x, \sigma, b_{\varphi}\right)>0$ for all $\sigma \in[\underline{\sigma}, \bar{\sigma}]$; and is $\bar{\sigma}$ if $Z\left(x, \sigma, b_{\varphi}\right)<0$ for all $\sigma \in[\underline{\sigma}, \bar{\sigma}]$.

The fictitious player chooses $\sigma^{\prime}$ to maximize

$$
\begin{aligned}
\int_{\underline{\sigma}}^{\bar{\sigma}} U_{n}\left(x, \sigma^{\prime}, \sigma_{0}\right) \varphi\left(d \sigma_{0}\right) & =\int_{\underline{\sigma}}^{\bar{\sigma}}\left(\int_{\underline{\sigma}}^{\sigma^{\prime}}-Z\left(x, s, b=p^{H}\left(\sigma_{0}\right)\right) d s\right) d \varphi\left(d \sigma_{0}\right) \\
& =\int_{\underline{\sigma}}^{\sigma^{\prime}}\left(\int_{\underline{\sigma}}^{\bar{\sigma}}-Z\left(x, s, b=p^{H}\left(\sigma_{0}\right)\right) d \varphi\left(d \sigma_{0}\right)\right) d s \\
& =\int_{\underline{\sigma}}^{\sigma^{\prime}}-Z\left(x, s, b_{\varphi}\right) d s .
\end{aligned}
$$

(The change of integration order in the second equality follows from standard arguments, while the third equality follows from the linearity of $Z$ in $b$.)

By prior arguments (see the proof of Lemma 1) we know that if $Z\left(x, \hat{\sigma}, b_{\varphi}\right)=0$ for some $\hat{\sigma}$, then $Z\left(x, \sigma, b_{\varphi}\right)<0$ for $\sigma<\hat{\sigma}$ and $Z\left(x, \sigma, b_{\varphi}\right)>0$ for $\sigma>\hat{\sigma}$. It follows that if $Z\left(x, \sigma_{n}^{*}, b_{\varphi}\right)=0$ then $\sigma_{n}^{*}$ is the unique maximizer of $\int_{\underline{\sigma}}^{\sigma^{\prime}}-Z\left(x, s, b_{\varphi}\right) d s$; if $Z\left(x, \sigma, b_{\varphi}\right)>0$ for all $\sigma \in[\underline{\sigma}, \bar{\sigma}]$ then the unique maximizer of $\int_{\underline{\sigma}}^{\sigma^{\prime}}-Z\left(x, s, b_{\varphi}\right) d s$ is $\underline{\sigma}$; and finally, if $Z\left(x, \sigma, b_{\varphi}\right)<0$ for all $\sigma \in[\underline{\sigma}, \bar{\sigma}]$ then the unique maximizer of $\int_{\underline{\sigma}}^{\sigma^{\prime}}-Z\left(x, s, b_{\varphi}\right) d s$ is $\bar{\sigma}$. This completes the proof. 
Proof of Proposition 1: As established in Lemma 2, it is possible to replace the $n$ voters with a single uninformed fictitious agent with preferences defined in (9). The proposer strictly prefers more acceptance (lower values of $\sigma_{n}^{*}$ ), regardless of his "type" $\sigma_{0}$ and offer $x$. Moreover, for any beliefs the best-response of the fictitious agent is a pure-strategy. As such, the game is strongly monotonic (see Manelli, page 929), and so possesses a sequential equilibrium (Manelli, Corollary 3).

Proof of Proposition 2: Differentiability follows directly from Lemma 1. For either $x<\underline{x}_{n}$ or $x>\bar{x}_{n}$, it is immediate that

$$
\frac{\partial}{\partial x} \Pi_{n}^{V}(x, b, \lambda, \alpha)=\frac{\partial}{\partial x} u_{i}\left(x, \sigma_{n}^{*}(x), b, \lambda, \alpha\right)
$$

since $\sigma_{n}^{*}(x)$ equals $\bar{\sigma}$ and $\underline{\sigma}$ respectively over these regions. For the intermediate case $x \in\left(\underline{x}_{n}, \bar{x}_{n}\right)$ we also need to account for the effect changing $x$ has on the equilibrium voting strategies. The main text handles the pure common values case $\lambda=0$. When $\alpha=1$ and $\lambda>0$, note that for any common cutoff strategy $\hat{\sigma}$

$$
\begin{aligned}
\frac{\partial}{\partial \hat{\sigma}_{i}} u_{i}(x, \hat{\sigma}, b, \lambda, \alpha) & =E_{\omega}\left[-f(\hat{\sigma} \mid \omega)(1-F(\hat{\sigma} \mid \omega))^{n-1} \Delta^{\omega}(x, \hat{\sigma})\right] \\
\frac{\partial}{\partial \hat{\sigma}_{j}} u_{i}(x, \hat{\sigma}, b, \lambda, \alpha) & =E_{\omega}\left[-f(\hat{\sigma} \mid \omega)(1-F(\hat{\sigma} \mid \omega))^{n-1} E\left[\Delta^{\omega}\left(x, \sigma_{i}\right) \mid \sigma_{i} \geq \hat{\sigma}\right]\right] .
\end{aligned}
$$

Since $E\left[\Delta^{\omega}\left(x, \sigma_{i}\right) \mid \sigma_{i} \geq \hat{\sigma}\right] \geq \Delta^{\omega}(x, \hat{\sigma})$ by Assumption 2, it follows that

$$
\frac{\partial}{\partial \hat{\sigma}_{i}} u_{i}(x, \hat{\sigma}, b, \lambda, \alpha) \geq \frac{\partial}{\partial \hat{\sigma}_{j}} u_{i}(x, \hat{\sigma}, b, \lambda, \alpha) .
$$

In equilibrium, (11) holds. Since $\partial \sigma_{n}^{*} / \partial x<0$ (see Lemma 1), the result then follows from (10).

Proof of Lemma 3: First, consider offers $x \geq x_{U}(b, \lambda)$. We claim that if $x_{U}(b, \lambda) \neq \infty$ then $\bar{x}_{n}(b, \lambda, \alpha=1)=x_{U}(b, \lambda)$ for all $n$. To see this, note that when $\alpha=1, Z(x, \underline{\sigma})$ coincides with the lefthand side of (12), regardless of $n$. As such, $Z\left(x_{U}, \underline{\sigma}\right)=0$, and so $Z\left(x_{U}, \sigma\right)>0$ for $\sigma>\underline{\sigma}$. So certainly $\bar{x}_{n} \geq x_{U}$. Moreover, it follows that $Z(x, \sigma)>0$ for all $\sigma$ if $x>x_{U}$, so that $\bar{x}_{n}=x_{U}$. Given this, the equilibrium for $x \geq x_{U}$ is the non-responsive acceptance equilibrium. 
Second, consider offers $x \in\left(x_{H}(\lambda), x_{U}(b, \lambda)\right)$. By definition, $\Delta^{H}\left(x_{H}(\lambda), \underline{\sigma}\right)=0$. It follows that for any $\sigma>0, Z\left(x_{H}(\lambda), \sigma\right)>0$ for $n$ large enough. So $\underline{x}_{n}(b, \lambda, \alpha) \leq x_{H}(\lambda)$ for $n$ large enough. As such, a responsive equilibrium exists whenever $n$ is large enough.

When a responsive equilibrium exists, the equilibrium condition is

$$
-\frac{\Delta^{H}\left(x, \sigma_{n}^{*}, \lambda\right)}{\Delta^{L}\left(x, \sigma_{n}^{*}, \lambda\right)} \frac{b}{1-b} \ell\left(\sigma_{n}^{*}\right)\left(\frac{1-F\left(\sigma_{n}^{*} \mid H\right)}{1-F\left(\sigma_{n}^{*} \mid L\right)}\right)^{n-1}=1 .
$$

Since $\frac{1-F(\sigma \mid H)}{1-F(\sigma \mid L)}>1$ for $\sigma>\underline{\sigma}$, it follows that

$$
\sigma_{n}^{*} \rightarrow \underline{\sigma} \text { as } n \rightarrow \infty
$$

In the proof of their Theorem 4, Duggan and Martinelli (2001) show that (A-1) and (A-2) together imply that

$$
\lim P_{n}^{H}=\lim \left(1-F\left(\sigma_{n}^{*} \mid H\right)\right)^{n}=\left(-\frac{\Delta^{H}(x, \underline{\sigma}, \lambda)}{\Delta^{L}(x, \underline{\sigma}, \lambda)} \frac{b}{1-b} \ell(\underline{\sigma})\right)^{\frac{f(\underline{\sigma} \mid H)}{f(\underline{\sigma} L)-f(\underline{\sigma} \mid H)}}
$$

i.e., equation (13) with $\omega=H$. The limit acceptance probability when $\omega=L$ then follows immediately from (A-1) and (A-2).

Third, consider the offer $x=x_{H}(\lambda)$, and suppose that contrary to the claimed result $\lim \left(1-F\left(\sigma_{n}^{*} \mid H\right)\right)^{n} \neq 0$. As such, there exists a subsequence of $\left(\sigma_{n}^{*}\right),\left(\sigma_{n_{m}}^{*}\right)$ say, such that $\lim _{m \rightarrow \infty}\left(1-F\left(\sigma_{n_{m}}^{*} \mid H\right)\right)^{n_{m}}>0$. Since $x_{H}(\lambda)<x_{U}(b, \lambda)$, for $m$ large $\sigma_{n_{m}}^{*}$ is a responsive equilibrium. As argued above, $\sigma_{n_{m}}^{*} \rightarrow \underline{\sigma}$. It follows that $\lim _{m \rightarrow \infty}\left(1-F\left(\sigma_{n_{m}}^{*} \mid H\right)\right)^{n_{m}}$ equals the righthand side of equation (A-3) evaluated at $x=x_{H}(\lambda)$. However, $-\frac{\Delta^{H}\left(x_{H}(\lambda), \underline{\sigma}, \lambda\right)}{\Delta^{L}\left(x_{H}(\lambda), \underline{\sigma}, \lambda\right)}=0$, and so this contradicts the hypothesis that $\lim _{m \rightarrow \infty}\left(1-F\left(\sigma_{n_{m}}^{*} \mid H\right)\right)^{n_{m}}>0$.

Fourth, and finally, if $x<x_{H}(\lambda)$ then from Corollary $1 P_{n}^{\omega}(x) \leq P_{n}^{\omega}\left(x_{H}(\lambda)\right)$ for all n. As such, $\lim P_{n}^{\omega}(x)=0$.

Finally, we prove continuity and monotonicity in $x$ and $b$. For $x$, observe that as its value increases from $x_{H}(\lambda)$ to $x_{U}(b, \lambda)<x_{L}(\lambda)$, the term $\Delta^{H}(x, \underline{\sigma}, \lambda)$ increases from 0 , and $\Delta^{L}(x, \underline{\sigma}, \lambda)$ increases and remains strictly negative. As such, the term inside parentheses in (A-3) increases from 0 , and equals 1 at $x=x_{U}(b, \lambda)$ (see definition 
(12)). For $b$, observe that if $x \leq x_{H}(\lambda)$, the limit acceptance probability is 0 for all $b$; while if $x \geq \sup _{b \in(0,1)} x_{U}(b, \lambda)$, the limit acceptance probability is 1 for all $b$. For the remaining case of $x \in\left(x_{H}(\lambda), \sup _{b \in(0,1)} x_{U}(b, \lambda)\right)$, continuity and monotonicity in $b$ are both immediate if $x<x_{U}(b, \lambda)$ for all $b \in(0,1)$. Otherwise, since $x_{U}(b, \lambda)$ is continuous and decreasing in $b$ there exists $\hat{b}$ such that $x=x_{U}(\hat{b}, \lambda), x<x_{U}(b, \lambda)$ if $b<\hat{b}$, and $x>x_{U}(b, \lambda)$ if $b>\hat{b}$. Monotonicity is then immediate, while continuity follows from $-\frac{\Delta^{H}(x, \underline{\sigma}, \lambda)}{\Delta^{L}(x, \underline{\sigma}, \lambda)} \frac{b}{1-b} \ell(\underline{\sigma})=1$ at $x=x_{U}(b, \lambda)$.

Before proving Lemma 4, we establish the following technical result which extends Helly's Selection Theorem from the real line to a compact Euclidian space under certain conditions.

Lemma A-2 Let $C=[a, b] \times D$, where $D$ is a compact Euclidian set, and let $g_{n}: C \rightarrow$ $\mathbb{R}$ be a sequence of continuous functions converging pointwise to a continuous function $g: C \rightarrow \mathbb{R}$. Suppose that $g_{n}$ is monotone in its first argument for all $n$, and has the following property:

There exists a compact Euclidean set $S$, a sequence of functions $s_{n}: C \rightarrow S$, and a continuous function $h: C \times S \rightarrow \mathbb{R}$ that is strictly monotone and continuously differentiable in its first argument such that, for all $n, g_{n}(z)=g_{n}\left(z^{\prime}\right)$ whenever $h\left(z, s_{n}(z)\right)=h\left(z^{\prime}, s_{n}(z)\right)$.

Then for any $\kappa>0, g_{n}$ converges uniformly to $g$ over $[a+\kappa, b-\kappa] \times D$.

Proof of Lemma A-2: Given $\kappa>0$, write $\hat{C}=[a+\kappa, b-\kappa] \times D$. Fix $\varepsilon>0$ and choose $\mu<\kappa$ such that $\left|g(z)-g\left(z^{\prime}\right)\right|<\frac{\varepsilon}{4}$ whenever $\left|z-z^{\prime}\right|<\mu$ and $z, z^{\prime} \in C$.

Define $\psi=\min _{(z, s) \in C \times S}\left|\frac{\partial h(z, s)}{\partial z_{1}}\right|$, and choose $\delta \in(0, \mu)$ such that $\delta<\psi \mu$.

Choose $\gamma \in(0, \mu)$ such that $\left|h(z, s)-h\left(z^{\prime}, s^{\prime}\right)\right|<\delta$ whenever $\left|(z, s)-\left(z^{\prime}, s^{\prime}\right)\right|<\gamma$ and $(z, s),\left(z^{\prime}, s^{\prime}\right) \in C \times S$.

Select a finite set $D^{*} \subset D$ such that for all $z_{-1} \in D$, there exists $z_{-1}^{\prime} \in D^{*}$ such that $\left|z_{-1}-z_{-1}^{\prime}\right|<\gamma$ 
By Helly's Selection Theorem, for any $z_{-1} \in D$ the function sequence $g_{n}\left(\cdot, z_{-1}\right)$ converges uniformly to $g\left(\cdot ; z_{-1}\right)$. So there exists some $N$ such that $\left|g_{n}\left(z_{1}, z_{-1}^{\prime}\right)-g\left(z_{1}, z_{-1}^{\prime}\right)\right|<$ $\frac{\varepsilon}{2}$ for any $\left(z_{1}, z_{-1}^{\prime}\right) \in[a, b] \times D^{*}$ whenever $n \geq N$.

Choose any $z \in \hat{C}$, and $n \geq N$. Let $z_{-1}^{\prime} \in D^{*}$ be such that $\left|z_{-1}-z_{-1}^{\prime}\right|<\gamma$. Note that

$$
h\left(z, s_{n}(z)\right) \in\left(h\left(z_{1}, z_{-1}^{\prime}, s_{n}(z)\right)-\delta, h\left(z_{1}, z_{-1}^{\prime}, s_{n}(z)\right)+\delta\right) .
$$

Moreover, as $\tilde{z_{1}}$ ranges over $\left(z_{1}-\mu, z_{1}+\mu\right)$ the value of $h\left(\tilde{z_{1}}, z_{-1}^{\prime}, s_{n}(z)\right)$ ranges continuously over a superset of

$$
\left(h\left(z_{1}, z_{-1}^{\prime}, s_{n}(y, z)\right)-\psi \mu, h\left(z_{1}, z_{-1}^{\prime}, s_{n}(y, z)\right)+\psi \mu\right) .
$$

Since $\delta<\psi \mu$, there exists some $z_{1}^{\prime} \in\left(z_{1}-\mu, z_{1}+\mu\right)$ such that

$$
h\left(z^{\prime}, s_{n}(z)\right)=h\left(z, s_{n}(z)\right)
$$

where $z^{\prime}=\left(z_{1}^{\prime}, z_{-1}^{\prime}\right) \in[a, b] \times D^{*}$. By assumption it follows that $g_{n}(z)=g_{n}\left(z^{\prime}\right)$, and so

$$
\begin{aligned}
\left|g_{n}(z)-g(z)\right| & \leq\left|g_{n}\left(z^{\prime}\right)-g\left(z^{\prime}\right)\right|+\left|g\left(z^{\prime}\right)-g\left(z_{1}, z_{-1}^{\prime}\right)\right|+\left|g\left(z_{1}, z_{-1}^{\prime}\right)-g(z)\right| \\
& \leq \frac{\varepsilon}{2}+\frac{\varepsilon}{4}+\frac{\varepsilon}{4}=\varepsilon .
\end{aligned}
$$

Proof of Lemma 4: The proof involves the application of Lemma A-2. To apply this result, define

$$
\begin{gathered}
D=[\underline{b}, \bar{b}] \times[0,1], \quad C=[0,1] \times D, \quad S=[\underline{\sigma}, \bar{\sigma}] \times[0,1], \\
h\left(x, b, \lambda, s^{1}, s^{2}\right)=b \Delta^{H}\left(x, s^{1}, \lambda\right) \ell\left(s^{1}\right) s^{2}+(1-b) \Delta^{L}\left(x, s^{1}, \lambda\right) \\
s_{n}(x, b, \lambda)=\left(\sigma_{n}^{*}(x, b, \lambda),\left(\frac{1-F\left(\sigma_{n}^{*}(x, b, \lambda) \mid H\right)}{1-F\left(\sigma_{n}^{*}(x, b, \lambda) \mid L\right)}\right)^{n-1}\right),
\end{gathered}
$$

and

$$
g_{n}(x, b, \lambda)=P_{n}^{\omega}(x, b, \lambda)
$$

Note that $h$ is strictly monotone and continuously differentiable in $x$. It remains to show that if $h\left(z, s_{n}(z)\right)=h\left(z^{\prime}, s_{n}(z)\right)$, then $g_{n}(z)=g_{n}\left(z^{\prime}\right)$. 
There are three cases to consider. First, suppose $\sigma_{n}^{*}(x, b, \lambda) \in(\underline{\sigma}, \bar{\sigma})$. By construction, $h\left(z, s_{n}(z)\right)=0$. Suppose that $h\left(z^{\prime}, s_{n}(z)\right)=0$, Then, by the uniqueness of responsive equilibrium, $\sigma_{n}^{*}\left(x^{\prime}, b^{\prime}, \lambda^{\prime}\right)=\sigma_{n}^{*}(x, b, \lambda)$.

For the next two cases, we claim that if $h_{n}\left(x, b, \lambda, \sigma,\left(\frac{1-F(\sigma \mid H)}{1-F(\sigma \mid L)}\right)^{n-1}\right) \geq 0$ at $\sigma=$ $\underline{\sigma}$, the same is true for all $\sigma \in[\underline{\sigma}, \bar{\sigma}]$; and likewise, if $h_{n}\left(x, b, \lambda, \sigma,\left(\frac{1-F(\sigma \mid H)}{1-F(\sigma \mid L)}\right)^{n-1}\right) \leq$ 0 at $\sigma=\bar{\sigma}$, the same is true for all $\sigma \in[\underline{\sigma}, \bar{\sigma}]$. Both these statements follow since $h_{n}\left(x, b, \lambda, \sigma,\left(\frac{1-F(\sigma \mid H)}{1-F(\sigma \mid L)}\right)^{n-1}\right)$ equals $Z(x, \sigma, b, \lambda, \alpha, n)$, and if $Z(x, \sigma, b, \lambda, \alpha, n) \geq 0$ for some $\sigma$, the same is true for all higher $\sigma$ (see proof of Lemma 1).

Now consider the case $\sigma_{n}^{*}(x, b, \lambda)=\underline{\sigma}$. By definition, $h_{n}\left(x, b, \lambda, \sigma,\left(\frac{1-F(\sigma \mid H)}{1-F(\sigma \mid L)}\right)^{n-1}\right) \geq$ 0 for all $\sigma$, and so in particular $h_{n}\left(x, b, \lambda, \underline{\sigma},\left(\frac{1-F(\underline{\sigma} \mid H)}{1-F(\underline{\sigma} \mid L)}\right)^{n-1}\right) \geq 0$. So if

$$
h_{n}\left(x^{\prime}, b^{\prime}, \lambda^{\prime}, \underline{\sigma},\left(\frac{1-F(\underline{\sigma} \mid H)}{1-F(\underline{\sigma} \mid L)}\right)^{n-1}\right)=h_{n}\left(x, b, \lambda, \underline{\sigma},\left(\frac{1-F(\underline{\sigma} \mid H)}{1-F(\underline{\sigma} \mid L)}\right)^{n-1}\right) \text {, }
$$

it follows (by above claim) that $h_{n}\left(x^{\prime}, b^{\prime}, \lambda^{\prime}, \sigma,\left(\frac{1-F(\sigma \mid H)}{1-F(\sigma \mid L)}\right)^{n-1}\right) \geq 0$ for all $\sigma$, and hence $\sigma_{n}^{*}\left(x^{\prime}, b^{\prime}, \lambda^{\prime}\right)=\underline{\sigma}$.

Finally, consider the case $\sigma_{n}^{*}(x, b, \lambda)=\bar{\sigma}$. By definition, $h_{n}\left(x, b, \lambda, \sigma,\left(\frac{1-F(\sigma \mid H)}{1-F(\sigma \mid L)}\right)^{n-1}\right) \leq$ 0 for all $\sigma$, and so in particular $h_{n}\left(x, b, \lambda, \bar{\sigma},\left(\frac{1-F(\overline{\underline{\sigma}} \mid H)}{1-F(\bar{\sigma} \mid L)}\right)^{n-1}\right) \leq 0$. So if

$$
h_{n}\left(x^{\prime}, b^{\prime}, \lambda^{\prime}, \bar{\sigma},\left(\frac{1-F(\bar{\sigma} \mid H)}{1-F(\bar{\sigma} \mid L)}\right)^{n-1}\right)=h_{n}\left(x, b, \lambda, \bar{\sigma},\left(\frac{1-F(\bar{\sigma} \mid H)}{1-F(\bar{\sigma} \mid L)}\right)^{n-1}\right) \text {, }
$$

it follows (by above claim) that $h_{n}\left(x^{\prime}, b^{\prime}, \lambda^{\prime}, \sigma,\left(\frac{1-F(\sigma \mid H)}{1-F(\sigma \mid L)}\right)^{n-1}\right) \leq 0$ for all $\sigma$, and hence $\sigma_{n}^{*}\left(x^{\prime}, b^{\prime}, \lambda^{\prime}\right)=\bar{\sigma}$.

Proof of Proposition 3: For clarity, we suppress $\alpha$ throughout. Let $v_{n}\left(x, \sigma_{0}, b, \lambda\right)$ denote the proposer's expected payoff from an offer $x$ when he has observed $\sigma_{0}$, the voters attach belief $b$ to offer $x$, and have preferences $\lambda$, i.e.,

$$
v_{n}\left(x, \sigma_{0}, b, \lambda\right) \equiv E_{\omega}\left[\bar{V}^{\omega}\left(\sigma_{0}\right)+P_{n}^{\omega}(x, b, \lambda)\left(V^{\omega}\left(x, \sigma_{0}\right)-\bar{V}^{\omega}\left(\sigma_{0}\right)\right) \mid \sigma_{0}\right]
$$

Define $P^{\omega}$ and $v$ as the pointwise limits of $P_{n}^{\omega}$ and $v_{n}$ respectively. 
We first establish that the proposer's offer is less than $x_{U}(\underline{b}, \lambda)$. If $x_{U}(\underline{b}, \lambda)=\infty$ this is vacuously true. If instead $x_{U}(\underline{b}, \lambda) \leq 1$, then $x_{U}(b, \lambda)<x_{U}(\underline{b}, \lambda)$ for all $b>\underline{b}$. From Lemma 3 , it follows that the offer $x_{U}(\underline{b}, \lambda)$ is accepted with probability one no matter what beliefs voters attach to it. As such, there is no equilibrium in which the proposer ever offers strictly more than $x_{U}(\underline{b}, \lambda)$.

Next, we establish the lower bound on the proposer's equilibrium offer. We know that for any beliefs $b, x_{H}(\lambda)<\min \left\{x_{U}(b, \lambda), 1\right\}$ at $\lambda=0$. Choose $\check{\lambda}>0$ and $\check{x}$ such that

$$
\max _{\lambda \in[0, \check{\lambda}]} x_{H}(\lambda)<\check{x}<\min _{\lambda \in[0, \check{\lambda}]} \min \left\{x_{U}(b, \lambda), 1\right\} .
$$

Choose $\varepsilon_{x}>0$ such that $\min _{\lambda \in[0, \check{\lambda}]} x_{H}(\lambda)>\varepsilon_{x}$ and $\check{x}<1-\varepsilon_{x}$.

From Lemma 3 , for any $(b, \lambda) \in[0, \check{\lambda}] \times[\underline{b}, \bar{b}]$ the limit acceptance probabilities $P^{\omega}(\check{x}, b, \lambda)$ are strictly positive for $\omega=L, H$. By continuity and compactness it follows that there exists $\varepsilon>0$ such that for any $\left(\sigma_{0}, b, \lambda\right) \in[\underline{\sigma}, \bar{\sigma}] \times[0, \check{\lambda}] \times[\underline{b}, \bar{b}]$,

$$
v\left(\check{x}, \sigma_{0}, b, \lambda\right)>E_{\omega}\left[\bar{V}^{\omega}\left(\sigma_{0}\right) \mid \sigma_{0}\right]+4 \varepsilon
$$

From Lemma $4, v_{n}$ converges uniformly to $v$ over $\left[\varepsilon_{x}, 1-\varepsilon_{x}\right] \times[\underline{\sigma}, \bar{\sigma}] \times[\underline{b}, \bar{b}] \times[0,1]$. This implies that there exists $N_{1}$ such that whenever $n \geq N_{1}$,

$$
v_{n}\left(\check{x}, \sigma_{0}, b, \lambda\right)>E_{\omega}\left[\bar{V}^{\omega}\left(\sigma_{0}\right) \mid \sigma_{0}\right]+3 \varepsilon \text {. }
$$

Let $\varpi=\max _{\omega, \sigma_{0}, x} V^{\omega}\left(x, \sigma_{0}\right)-\bar{V}^{\omega}\left(\sigma_{0}\right)$. From Lemma 3, there exists $\kappa>0$ such that $P^{\omega}(x, b, \lambda) \leq \frac{\varepsilon}{\varpi}$ if $x \in\left[0, x_{H}(\lambda)+\kappa\right]$. Uniform convergence of $P_{n}^{\omega}(\cdot)$ to $P^{\omega}(\cdot)$ (Lemma 4) implies that there exists $N_{2}$ such that $P_{n}^{\omega}\left(x_{H}(\lambda)+\kappa, b, \lambda\right) \leq \frac{2 \varepsilon}{\varpi}$ whenever $n \geq N_{2}$. Combined with monotonicity of the acceptance probability in the offer $x$, it follows that if $x \leq x_{H}(\lambda)+\kappa$ and $n \geq N_{2}$,

$$
v_{n}\left(x, \sigma_{0}, b, \lambda\right) \leq E_{\omega}\left[\bar{V}^{\omega}\left(\sigma_{0}\right) \mid \sigma_{0}\right]+2 \varepsilon
$$

Hence for $n \geq \max \left\{N_{1}, N_{2}\right\}$, for any $\left(\sigma_{0}, \lambda\right) \in[\underline{\sigma}, \bar{\sigma}] \times[0, \check{\lambda}]$ it cannot be an equilibrium for the proposer to offer $x \in\left[0, x_{H}(\lambda)+\kappa\right]$ : doing so generates at most $2 \varepsilon$ over the status 
quo payoff, while offering $\check{x}$ generates at least $3 \varepsilon$ over the status quo payoff, regardless of beliefs. This completes the proof.

Proof of Lemma 5: We prove the lemma in four steps. For clarity, we suppress $\lambda$ and $\alpha$ and write $x_{\omega}$ in place of $x_{\omega}(\lambda ; \alpha)$ throughout.

Claim 1 If $\lim \sup x_{n}<x_{H}$ then $\liminf \sigma_{n}^{*}>\sigma_{H}$.

Proof: By hypothesis, there exists $\varepsilon$ such that $x_{n} \leq x_{H}-\varepsilon$ for all $n$ large enough. Suppose that contrary to the claim, $\liminf \sigma_{n}^{*} \leq \sigma_{H}$. So for any $\delta>0$, there exists a subsequence of $\sigma_{n}^{*}$ such that $\sigma_{n}^{*} \leq \sigma_{H}+\delta$. By definition $\Delta^{H}\left(x_{H}, \sigma_{H}, \lambda\right)=0$; so for $\delta$ small enough, there exists $\hat{\varepsilon}$ such that $\Delta^{H}\left(x_{n}, \sigma_{n}^{*}, \lambda\right)<-\hat{\varepsilon}$. Moreover, $\Delta^{L}\left(x_{n}, \sigma_{n}^{*}, \lambda\right) \leq \Delta^{H}\left(x_{n}, \sigma_{n}^{*}, \lambda\right)$. Consequently $Z\left(x_{n}, \sigma_{n}^{*}\right)<0$. As such, $\sigma_{n}^{*}$ is not a responsive equilibrium; and since $x_{n} \leq \bar{x}_{n}$ then $\sigma_{n}^{*}$ is not an acceptance equilibrium either. The only remaining possibility is that $\sigma_{n}^{*}$ is a rejection equilibrium - but then $\sigma_{n}^{*}=\bar{\sigma}$, which gives a contradiction when $\delta$ is chosen small enough.

Claim 2 If $\lim \sup x_{n}<x_{L}$ then $\lim \inf \sigma_{n}^{*}>\sigma_{L}$.

Proof: By hypothesis, there exists $\varepsilon$ such that $x_{n} \leq x_{L}-\varepsilon$ for all $n$ large enough. Suppose that contrary to the claim, $\lim \inf \sigma_{n}^{*} \leq \sigma_{L}$. So for any $\delta>0$, there exists a subsequence of $\sigma_{n}^{*}$ such that $\sigma_{n}^{*} \leq \sigma_{L}+\delta$. By definition $\Delta^{L}\left(x_{L}, \sigma_{L}, \lambda\right)=0$; so for $\delta$ small enough, there exists $\hat{\varepsilon}$ such that $\Delta^{L}\left(x_{n}, \sigma_{n}^{*}, \lambda\right)<-\hat{\varepsilon}$. Next, define

$$
\phi=\max _{\sigma \in\left[\underline{\sigma}, \sigma_{L}+\delta\right]} \frac{(1-F(\sigma \mid H))^{\alpha} F(\sigma \mid H)^{1-\alpha}}{(1-F(\sigma \mid L))^{\alpha} F(\sigma \mid L)^{1-\alpha}}
$$

Note that the function $(1-q)^{\alpha} q^{1-\alpha}$ is increasing for $q \in(0,1-\alpha)$ and decreasing for $q \in(1-\alpha, 1)$. Recall that by definition $F\left(\sigma_{L} \mid L\right)=1-\alpha$, and by Lemma A-1 $F(\sigma \mid H)<$ $F(\sigma \mid L)$ for all $\sigma \in(\underline{\sigma}, \bar{\sigma})$. It follows that $\phi<1$ for $\delta$ chosen small enough, and so

$$
\left(\frac{\left(1-F\left(\sigma^{*} \mid H\right)\right)^{\alpha} F\left(\sigma^{*} \mid H\right)^{1-\alpha}}{\left(1-F\left(\sigma^{*} \mid L\right)\right)^{\alpha} F\left(\sigma^{*} \mid L\right)^{1-\alpha}}\right)^{n} \leq \phi^{n} \rightarrow 0
$$


Since $\sigma_{n}^{*}$ is bounded away from $\bar{\sigma}$, then $1-F\left(\sigma_{n}^{*} \mid H\right)$ is bounded away from 0 . By belief consistency, $\frac{\beta_{n}\left(x_{n}\right)}{1-\beta_{n}\left(x_{n}\right)}$ is bounded away from infinity. Consequently $Z\left(x_{n}, \sigma_{n}^{*}\right)<0$ for $n$ sufficiently large. A contradiction then follows as in Claim 1.

Claim 3 If $\lim \inf x_{n}>x_{L}$ then $\lim \sup \sigma_{n}^{*}<\sigma_{L}$.

Claim 4 If $\lim \inf x_{n}>x_{H}$ then $\lim \sup \sigma_{n}^{*}<\sigma_{H}$.

Proofs of Claims 3 and 4: Exactly parallel to those of Claims 1 and 2.

Proof of Lemma 6: We focus on Part 1a. (Part $1 \mathrm{~b}$ and 2 are proved by similar arguments, which we omit for conciseness. Part 3 is immediate from Lemma 5.) The main idea is straightforward: for any $\sigma_{0}$ such that $W\left(\sigma_{0}\right)>0$, the proposer prefers offering $x_{H}(\lambda ; \alpha)$ and gaining acceptance if and only if $\omega=H$ to offering $x_{L}(\lambda, \alpha)$ and gaining acceptance all the time. Given the limiting behavior of voters established in Lemma 5 , intuitively it follows that the proposer's offer converges to $x_{H}(\lambda ; \alpha)$ as the number of voters grows large. The main difficulty encountered in the formal proof is establishing uniform convergence: for any $\varepsilon, \delta>0$, there is some $N(\varepsilon, \delta)$ such that when $n \geq N(\varepsilon, \delta)$, the proposer's offer lies within $\delta$ of $x_{H}(\lambda ; \alpha)$ for all $\sigma_{0}$ such that $W\left(\sigma_{0}\right)>\varepsilon$.

Take any $\varepsilon, \delta>0$. Throughout the proof, we omit all $\lambda$ and $\alpha$ arguments for readability. We define $\Delta_{0}^{\omega}\left(x, \sigma_{0}\right) \equiv V^{\omega}\left(x, \sigma_{0}\right)-\bar{V}^{\omega}\left(\sigma_{0}\right)$, the proposer's gain to offer $x$ being accepted conditional on $\omega$.

Preliminaries: The first part of the proof consists of defining bounds which we will use to establish uniform convergence below. Choose $\mu, \delta_{1}, \delta_{2}, \delta_{3} \in(0, \delta]$ such that $x_{H}+\mu<x_{L}-\mu$, and for all $\sigma_{0}$ for which $W\left(\sigma_{0}\right)>\varepsilon$,

$$
\begin{gathered}
p^{H}\left(\sigma_{0}\right) V^{H}\left(x_{H}+\frac{\mu}{2}, \sigma_{0}\right)+p^{L}\left(\sigma_{0}\right) \bar{V}^{L}\left(\sigma_{0}\right) \geq E\left[V^{\omega}\left(x_{L}-\mu, \sigma_{0}\right) \mid \sigma_{0}\right]+\frac{\varepsilon}{2}, \\
\delta_{1} \Delta_{0}^{H}\left(x_{H}+\frac{\mu}{2}, \sigma_{0}\right) \leq \frac{\varepsilon}{4}, \\
\delta_{2}\left(p^{H}\left(\sigma_{0}\right) \Delta_{0}^{H}\left(0, \sigma_{0}\right)+p^{L}\left(\sigma_{0}\right) \Delta_{0}^{L}\left(0, \sigma_{0}\right)\right)<\left(1-\delta_{1}\right) p^{H}\left(\sigma_{0}\right) \Delta_{0}^{H}\left(x_{H}+\frac{\mu}{2}, \sigma_{0}\right),
\end{gathered}
$$




$$
\begin{aligned}
& p^{H}\left(\sigma_{0}\right)\left(\left(1-\delta_{1}\right) \Delta_{0}^{H}\left(x_{H}+\frac{\mu}{2}, \sigma_{0}\right)-\Delta_{0}^{H}\left(x_{H}+\mu, \sigma_{0}\right)\right)>p^{L}\left(\sigma_{0}\right) \delta_{3} \Delta_{0}^{L}\left(x_{H}+\mu, \sigma_{0}\right), \\
& p^{H}\left(\sigma_{0}\right)\left(\left(1-\delta_{1}\right) V^{H}\left(x_{H}+\frac{\mu}{2}, \sigma_{0}\right)+\delta_{1} \bar{V}^{H}\left(\sigma_{0}\right)\right)+p^{L}\left(\sigma_{0}\right) \bar{V}^{L}\left(\sigma_{0}\right) \\
& >p^{H}\left(\sigma_{0}\right)\left((1-\delta) V^{H}\left(x_{H}-\mu, \sigma_{0}\right)+\delta \bar{V}^{H}\left(\sigma_{0}\right)\right) \\
& +p^{L}\left(\sigma_{0}\right)\left(\delta_{3} V^{L}\left(x_{H}-\mu, \sigma_{0}\right)+\left(1-\delta_{3}\right) \bar{V}^{L}\left(\sigma_{0}\right)\right) .
\end{aligned}
$$

A choice of $\mu, \delta_{1}, \delta_{2}, \delta_{3}$ exists such that (A-5), (A-6), (A-7), (A-8), and (A-9) hold as follows. First, choose $\mu$ such that (A-5) holds, along with

$$
V^{H}\left(x_{H}+\frac{\mu}{2}, \sigma_{0}\right)>(1-\delta) V^{H}\left(x_{H}-\mu, \sigma_{0}\right)+\delta \bar{V}^{H}\left(\sigma_{0}\right) .
$$

It is possible to choose $\mu>0$ that satisfies these two inequalities for all $\sigma_{0}$ since $\left|V_{x}^{\omega}\right|$ is bounded. The same argument applies in choosing $\delta_{1}, \delta_{2}, \delta_{3}$ below. Second, choose $\delta_{1}$ such that (A-6) holds, along with

$$
\begin{gathered}
\left(1-\delta_{1}\right) \Delta_{0}^{H}\left(x_{H}+\frac{\mu}{2}, \sigma_{0}\right)-\Delta_{0}^{H}\left(x_{H}+\mu, \sigma_{0}\right)>0, \\
\left(\left(1-\delta_{1}\right) V^{H}\left(x_{H}+\frac{\mu}{2}, \sigma_{0}\right)+\delta_{1} \bar{V}^{H}\left(\sigma_{0}\right)\right)-\left((1-\delta) V^{H}\left(x_{H}-\mu, \sigma_{0}\right)+\delta \bar{V}^{H}\left(\sigma_{0}\right)\right)>0,
\end{gathered}
$$

where (A-12) is possible by (A-10). Third, choose $\delta_{2}$ such that (A-7) holds. Finally, choose $\delta_{3}$ such that (A-8) and (A-9) hold, which is possible by (A-11) and (A-12) respectively.

Let $\underline{b}$ and $\bar{b}$ respectively denote the most pro- $L$ and pro- $H$ beliefs possible. Fix a realization of $\sigma_{0}$ such that $W\left(\sigma_{0}\right) \geq \varepsilon$. Define the following offer sequences, which we use throughout the proof:

$$
x_{n}^{H+} \equiv x_{H}+\frac{\mu}{2}, \quad x_{n}^{H-} \equiv x_{H}-\mu, \quad x_{n}^{L-} \equiv x_{L}-\mu .
$$

By Lemma $5, P_{n}^{H}\left(x_{n}^{H+}, \underline{b}\right) \rightarrow 1$ and $P_{n}^{L}\left(x_{n}^{H+}, \bar{b}\right) \rightarrow 0 ; P_{n}^{\omega}\left(x_{n}^{H-}, \bar{b}\right) \rightarrow 0$ for $\omega=L, H$; and $P_{n}^{L}\left(x_{n}^{L-}, \bar{b}\right) \rightarrow 0$. Thus there exist $N_{1}, N_{2}, N_{3}$ such that $P_{n}^{H}\left(x_{n}^{H+}, \underline{b}\right) \geq 1-\delta_{1}$ and $P_{n}^{L}\left(x_{n}^{H+}, \bar{b}\right) \leq \delta_{1}$ for $n \geq N_{1} ; P_{n}^{\omega}\left(x_{n}^{H-}, \bar{b}\right) \leq \delta_{2}$ for $\omega=L, H$ and $n \geq N_{2}$; and 
$P_{n}^{L}\left(x_{n}^{L-}, \bar{b}\right) \leq \delta_{3}$ for $n \geq N_{3}$. Let $N(\varepsilon, \delta)=\max \left\{N_{1}, N_{2}, N_{3}\right\}$. Note that $N(\varepsilon, \delta)$ depends only on $\varepsilon$ and $\delta$, and not $\sigma_{0}$.

Given $\sigma_{0}$, choose $x_{n}$ from the set of equilibrium offers when there are $n$ voters.

Part A: If $W\left(\sigma_{0}\right) \geq \varepsilon$ and $n \geq N(\varepsilon, \delta)$, then for any equilibrium offer $x_{n}, P_{n}^{L}\left(x_{n}\right) \leq$ $\delta_{3} \leq \delta$

Proof: If $x_{n} \leq x_{n}^{L-}$ then $P_{n}^{L}\left(x_{n}\right) \leq P_{n}^{L}\left(x^{L-}, \bar{b}\right) \leq \delta_{3}$ for $n \geq N(\varepsilon, \delta)$. Consequently it suffices to show that $x_{n} \leq x_{n}^{L-}$ for all $n \geq N(\varepsilon, \delta)$. If this were not the case, there must exist some $m \geq N(\varepsilon, \delta)$ such that $x_{m}>x_{m}^{L-}$. By Assumption 4 the proposer is always better off when his offer is accepted; and so if $x_{m}>x_{m}^{L-}$ the proposer's expected payoff is bounded above by $E\left[V^{\omega}\left(x_{m}^{L-}, \sigma_{0}\right) \mid \sigma_{0}\right]$. In contrast, since $m \geq N(\varepsilon, \delta)$, the proposer's payoff from the offer $x_{m}^{H+}$ is bounded below by

$$
\begin{aligned}
& p^{H}\left(\sigma_{0}\right)\left(\left(1-\delta_{1}\right) V^{H}\left(x_{m}^{H+}, \sigma_{0}\right)+\delta_{1} \bar{V}^{H}\left(\sigma_{0}\right)\right)+p^{L}\left(\sigma_{0}\right) \bar{V}^{L}\left(\sigma_{0}\right) \\
= & p^{H}\left(\sigma_{0}\right)\left(V^{H}\left(x_{m}^{H+}, \sigma_{0}\right)-\delta_{1} \Delta_{0}^{H}\left(x_{m}^{H+}, \sigma_{0}\right)\right)+p^{L}\left(\sigma_{0}\right) \bar{V}^{L}\left(\sigma_{0}\right) \\
\geq & p^{H}\left(\sigma_{0}\right) V^{H}\left(x_{m}^{H+}, \sigma_{0}\right)+p^{L}\left(\sigma_{0}\right) \bar{V}^{L}\left(\sigma_{0}\right)-\frac{\varepsilon}{4}
\end{aligned}
$$

where the inequality follows by $(\mathrm{A}-6)$ (and the fact that $p^{H}\left(\sigma_{0}\right) \leq 1$ ). By $(\mathrm{A}-5)$ this lower bound exceeds $E\left[V^{\omega}\left(x_{m}^{L-}, \sigma_{0}\right) \mid \sigma_{0}\right]$, contradicting the optimality of $x_{n}$.

Part B: If $W\left(\sigma_{0}\right) \geq \varepsilon$ and $n \geq N(\varepsilon, \delta)$, then for any equilibrium offer $x_{n},\left|x_{n}-x_{H}\right| \leq$ $\mu \leq \delta$

Proof: First, we claim that $x_{n}>x_{n}^{H-}$ whenever $n \geq N(\varepsilon, \delta)$. If this were not the case, there must exist some $m \geq N(\varepsilon, \delta)$ such that $x_{m} \leq x_{m}^{H-}$. The acceptance probability of $x_{m}$ given $\omega$ is consequently less than that of $x_{m}^{H-}$ under the most pro-acceptance beliefs $\bar{b}$, which is in turn less than $\delta_{2}$. The acceptance probability of $x_{m}^{H+}$ given $H$ is at least $1-\delta_{1}$. It follows from (A-7) that the proposer's payoff is higher under $x_{m}^{H+}$ than under $x_{m}$. But this contradicts the optimality of the proposer's offer $x_{m}$. Second, we claim that $x_{n} \leq x_{H}+\mu$ whenever $n \geq N(\varepsilon, \delta)$. If not, there exists $m \geq N(\varepsilon, \delta)$ such that $x_{m}>x_{H}+\mu$. By Part A, proposer's payoff under $x_{m}$ is bounded above by

$$
p^{H}\left(\sigma_{0}\right)\left(\Delta_{0}^{H}\left(x_{H}+\mu, \sigma_{0}\right)+\bar{V}^{H}\left(\sigma_{0}\right)\right)+p^{L}\left(\sigma_{0}\right)\left(\delta_{3} \Delta_{0}^{L}\left(x_{H}+\mu, \sigma_{0}\right)+\bar{V}^{L}\left(\sigma_{0}\right)\right) .
$$


In contrast, since $m \geq N(\varepsilon, \delta)$, the proposer's payoff from the offer $x_{m}^{H+}$ is bounded below by

$$
p^{H}\left(\sigma_{0}\right)\left(\left(1-\delta_{1}\right) \Delta_{0}^{H}\left(x_{m}^{H+}, \sigma_{0}\right)+\bar{V}^{H}\left(\sigma_{0}\right)\right)+p^{L}\left(\sigma_{0}\right) \bar{V}^{L}\left(\sigma_{0}\right),
$$

which exceeds the payoff from the offer $x_{m}$ by (A-8), contradicting optimality of $x_{m}$.

Part C: If $W\left(\sigma_{0}\right) \geq \varepsilon$ and $n \geq N(\varepsilon, \delta)$, then for any equilibrium offer $x_{n}, P_{n}^{H}\left(x_{n}\right) \geq$ $1-\delta$.

Proof: Suppose that contrary to the claim, there exists $m \geq N(\varepsilon, \delta)$ such that $P_{m}^{H}\left(x_{m}\right)<$ $1-\delta$. By Part A, $P_{n}^{L}\left(x_{m}^{L-}, \bar{b}\right) \leq \delta_{3}$, and by Part B, $x_{m} \geq x_{m}^{H-}$ and hence proposer's payoff is bounded above by

$p^{H}\left(\sigma_{0}\right)\left((1-\delta) V^{H}\left(x_{m}^{H-}, \sigma_{0}\right)+\delta \bar{V}^{H}\left(\sigma_{0}\right)\right)+p^{L}\left(\sigma_{0}\right)\left(\delta_{3} V^{L}\left(x_{m}^{H-}, \sigma_{0}\right)+\left(1-\delta_{3}\right) \bar{V}^{L}\left(\sigma_{0}\right)\right)$.

In contrast, under the offers $x_{m}^{H+}$, the proposer's payoff is bounded below by

$$
p^{H}\left(\sigma_{0}\right)\left(\left(1-\delta_{1}\right) V^{H}\left(x_{m}^{H+}, \sigma_{0}\right)+\delta_{1} \bar{V}^{H}\left(\sigma_{0}\right)\right)+p^{L}\left(\sigma_{0}\right) \bar{V}^{L}\left(\sigma_{0}\right) .
$$

By (A-9) the latter is strictly greater, contradicting the optimality of the offers $x_{n}$. 